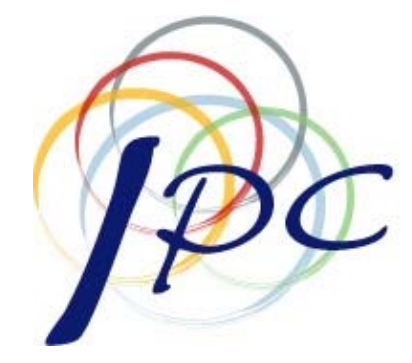

International Policy Center

Gerald R. Ford School of Public Policy University of Michigan

IPC Working Paper Series Number 83

Prospect Theory and Market Liquidity

Paolo Pasquariello

October 28, 2008 


\title{
Prospect Theory and Market Liquidity
}

\author{
Paolo Pasquariello ${ }^{1}$
}

October 28,2008

\footnotetext{
${ }^{1}$ The author is affiliated with the department of Finance at the Ross School of Business, University of Michigan. Please address comments to the author via email at ppasquar@bus.umich.edu. I benefited from the comments of Nick Barberis, Sreedhar Bharath, David Hirshleifer, Bruce Lehmann, Amiyatosh Purnanandam, Uday Rajan, Wei Xiong, and seminar participants at the University of Michigan. Any remaining errors are my own.
} 


\begin{abstract}
We study equilibrium trading strategies, market liquidity, and price efficiency in an economy in which a fraction of better-informed speculators displays preferences consistent with Kahneman and Tversky's (1979) Prospect Theory, i.e., loss aversion, risk seeking over losses, and nonlinear and asymmetric probability weighting (in the spirit of Jullien and Salanié, 2000). Loss aversion induces those speculators to trade more cautiously, while risk seeking induces them to trade more aggressively, with their private signals. We demonstrate that the latter effect dominates the former in equilibrium, leading to lower and (because of procyclical subjective loss probabilities) countercyclical adverse selection-based market liquidity and higher price efficiency. We also find that the presence of those speculators affects the extent to which the release of public news about the traded asset's terminal payoff improves market liquidity and price efficiency and makes such improvement procyclical.
\end{abstract}

JEL classification: D82; G14

Keywords: Prospect Theory; Market Liquidity; Price Efficiency; Adverse Selection; Loss Aversion; Risk Seeking; Probability Weighting 


\section{Introduction}

Over the past two decades, a large and long-standing body of experimental evidence on human behavior has provided support to the notion, first formulated by Kahneman and Tversky (1979) as Prospect Theory, that the decision-making process of economic agents may depart from the predictions of standard expected utility theory. Prospect Theory proposes that those agents assess gambles with a value function defined over gains and losses relative to a reference point (instead of the absolute level of financial wealth or consumption), concave over gains (risk aversion), but convex (risk seeking) and steeper (loss aversion) over losses. Prospect Theory also suggests that, in assessing gambles, economic agents employ nonlinear and asymmetric transformations of the objective cumulative probability distribution of payoffs overweighting its tails. In particular, Jullien and Salanié (2000) find that those agents are disproportionately afraid of the low probability of a loss when selecting gambles with high probability of a gain. Recent work employs modified versions of this theory to study the pricing of financial securities. Prospect Theory arguments have been used to explain such known asset pricing puzzles as the magnitude of the equity premium, excess stock return volatility, momentum and the disposition effect, the value premium, or stock return predictability and its implications for portfolio selection. ${ }^{1}$

The past two decades have also been characterized by an increasing interest in the study of the process of price formation in financial markets. A voluminous literature known as Market Microstructure, fueled by the vast amount of information that these markets now produce and make available to researchers, has studied (both theoretically and empirically) such issues as the mechanisms through which private and common information are impounded into prices,

\footnotetext{
${ }^{1}$ E.g., Benartzi and Thaler (1995), Aït-Sahalia and Brandt (2001), Barberis, Huang, and Santos (2001), Barberis and Huang (2001), Berkelaar, Kouwenberg, and Post (2004), Gomes (2005), Grinblatt and Han (2005), Barberis, Huang, and Thaler (2006), Barberis and Xiong (2006), Kyle, Ou-Yang, and Xiong (2006), and Barberis and Huang (2008).
} 
agents' reasons for trade and optimal trading strategies, and liquidity. ${ }^{2}$ Yet, to our knowledge, this literature has not examined any of these issues when investors make decisions according to Prospect Theory.

The main objective of this paper is to investigate formally the effect of Prospect Theory on market liquidity and price efficiency. We start by describing the equilibrium of a benchmark one-period model of trading — in the spirit of Kyle (1985) and Subrahmanyam (1991) but also Grossman and Stiglitz (1980) — populated by competitive, informed agents with an exponential utility function (heretofore MV speculators), noise traders, and competitive market-making (heretofore MM). The benchmark model's implications for market depth, trading strategies, and the informational efficiency (informativeness) of prices are standard in the literature. We then introduce informed agents (heretofore PT speculators) with preferences capturing all of the main features of Prospect Theory mentioned above parsimoniously while allowing for a noisy rational expectations equilibrium of the economy in closed form. ${ }^{3}$

The ensuing analysis makes two related contributions to the literature. First, it generates several novel predictions for market depth and price informativeness that hinge upon the presence of speculators motivated by Prospect Theory-inspired preferences. Second, the predictions it documents are testable, thus possibly refutable, rather than aimed at matching extant features of the data. As such, they provide an unbiased, albeit more challenging opportunity for researchers to assess the empirical relevance of unconventional utility models.

We show that the presence of PT speculators worsens a traded risky asset's adverse selectionbased market liquidity and improves its price informativeness in equilibrium. Intuitively, loss

\footnotetext{
${ }^{2}$ E.g., see the surveys of O'Hara (1995) and Hasbrouck (2007).

${ }^{3}$ Barberis and Huang (2008) study the asset pricing implications of Tversky and Kahneman's (1992) cumulative Prospect Theory utility model, including its probability weighting component. Kyle, Ou-Yang, and Xiong (2006) abstract from the latter when examining an agent's decision whether to liquidate an asset before its natural payoff. Previous research (e.g., Barberis, Huang, and Santos, 2001; Barberis and Huang, 2001) concentrates exclusively on agents' greater sensitivity to reductions of their financial wealth.
} 
aversion induces PT speculators to more cautious trading with their private signal, the more so the greater is their marginal loss probability; risk seeking induces PT speculators to more aggressive trading with their private signal, the more so the greater is their subjective (i.e., transformed) cumulative loss probability. In equilibrium, the latter effect dominates the former, and PT speculators trade more aggressively than the otherwise identical MV speculators. Such additional aggressiveness makes the order flow more informative about the risky asset's fundamentals and the MM's adverse selection problem more severe. ${ }^{4}$

We also show that the presence of PT speculators makes average equilibrium adverse selectionbased market depth countercyclical, i.e., lower during "good" times (positive private signals) than during "bad" times (negative private signals). An intuitive explanation for this finding is that ceteris paribus, PT speculators' subjective cumulative loss probability is higher (and their riskseeking behavior more intense) the greater is the private signal they observe (and the price at which they trade) - i.e., is procyclical — which leads to greater trading aggressiveness and worse market liquidity. Consistently, recent empirical evidence suggests that market and stock-level liquidity may be state-dependent (and possibly countercyclical), especially that of smaller firms, where PT speculation may play a bigger role (e.g., Chordia, Roll, and Subrahmanyam, 2001; Chordia, Shivakumar, and Subrahmanyam, 2004; Griffin, Nardari, and Stulz, 2007; Kamara, Lou, and Sadka, 2007; Deuskar, 2008). Nevertheless, these papers do not directly test whether those dynamics can be attributed to the presence and trading activity of PT speculators.

The econometrician cannot observe speculators' private signals when estimating traded assets' market depth and price efficiency. However, public information about traded assets' fundamentals is frequently released to all market participants, typically improving both (e.g., Pasquariello and Vega, 2007). We therefore extend our model to consider whether the presence of PT speculators affects the impact of such a release on a traded asset's process of price formation. The amended

\footnotetext{
${ }^{4}$ Consistently, Kyle, Ou-Yang, and Xiong (2006) show that the liquidation decision of economic agents with Prospect Theory-inspired preferences is driven primarily by the convexity in their value function.
} 
model generates a rich set of state-dependent comparative statics. In particular, we find that the extent of that improvement is sensitive to the fraction of PT speculators in the economy and greater the better public news is released and/or the better private news is available (i.e., is procyclical), because those circumstances affect the informativeness of the aggregate order flow and the severity of the MM's perceived adverse selection risk.

Our work is related to Subrahmanyam (1991) and Foster and Viswanathan (1993). Subrahmanyam (1991) allows for noncompetitive, risk-averse speculators in the one-period noisy rational expectations model of Kyle (1985). In this paper we abstract from imperfect competition to concentrate on the implications of Prospect Theory preferences for liquidity and price efficiency. Unreported analysis indicates that our intuition would be basically immune from such an extension. In addition, we show that our benchmark model is able to replicate the main comparative statics in Subrahmanyam (1991). In a similar setting, Foster and Viswanathan (1993) demonstrate that price impact and the variance of prices are state-dependent when representing speculators' beliefs with elliptically contoured distributions. However, there is little or no evidence guiding the analyst's modelling choice for those unobservable beliefs. In our model state-dependency ensues from state-dependent, Prospect Theory-inspired preferences even when all random variables are normally distributed. Another related literature explores asset pricing implications of investors exhibiting either irrationality or bounded rationality. ${ }^{5}$ All agents in our model, including the fraction of better-informed speculators displaying nonconventional preferences, are instead fully rational.

We proceed as follows. In Section 2, we construct a benchmark model of trading. In Section 3, we introduce PT speculators and discuss the implications of their presence for market liquidity and price efficiency. In Section 4, we enrich the model by endowing each agent with a public signal of the traded asset's liquidation value. We conclude in Section 5.

\footnotetext{
${ }^{5}$ E.g., Barberis, Schleifer, and Vishny (1998), Hong and Stein (1999), Daniel, Hirshleifer, and Subrahmanyam (2001), and Kogan, Ross, Wang, and Westerfield (2006).
} 


\section{The Benchmark Model}

In this section we describe a benchmark noisy rational expectations model of trading in the presence of better-informed, risk-averse agents and derive closed-form solutions for the equilibrium trading strategies, adverse selection-based market depth, and price efficiency. The model's structure is similar to Kyle (1985) and Subrahmanyam (1991); yet, we assume that those agents take the equilibrium prices as given, in the spirit of Grossman and Stiglitz (1980), Diamond and Verrecchia (1981), and Verrecchia (1982). This assumption is made solely for simplicity. Allowing for imperfect competition complicates the analysis that follows considerably without significantly affecting its main intuition. ${ }^{6}$ The benchmark model's implications for market liquidity and price informativeness are standard. In the next section we enrich this basic structure by introducing informed agents with Prospect Theory-inspired preferences and consider the properties of the ensuing equilibrium. All proofs are in the Appendix unless otherwise noted.

\subsection{Risk-Averse Speculators}

The basic model is a two-date, one-period economy in which a single risky asset is exchanged. Trading occurs only at the end of the period $(t=1)$, after which the asset payoff, $v$, is realized. The economy is populated by three types of traders: A continuum of risk-averse informed traders (that we label speculators) with a total mass of one, liquidity traders, and a competitive, riskneutral market-maker (MM). ${ }^{7}$ All traders know the structure of the economy and the decision

\footnotetext{
${ }^{6}$ For instance, when the risk-averse informed agents are assumed to be imperfectly competitive, a closedform expression for the equilibrium market liquidity cannot be obtained, as in Subrahmanyam (1991). Further, Kovalenkov and Vives (2007) and Vives (2008) show that in the presence of risk-averse informed traders, a pricetaking rational expectations equilibrium provides a reasonably close approximation to the corresponding strategic equilibrium.

${ }^{7}$ For an analysis of risk-averse market-making in an adverse selection model à la Kyle (1985), see Subrahmanyam (1991).
} 
process leading to order flow and prices.

At time $t=0$ there is neither information asymmetry about $v$ nor trading. Sometime between $t=0$ and $t=1$, all speculators receive a private and noisy signal of $v, S=v+u$. The random variables $v$ and $u$ are assumed to be mutually independent and normally distributed with mean zero and variance $\sigma_{v}^{2}$ and $\sigma_{u}^{2}$, respectively. It then ensues that $\operatorname{var}[S] \equiv \sigma_{s}^{2}=\sigma_{v}^{2}+\sigma_{u}^{2}$ and $\operatorname{cov}[v, S]=\sigma_{v}^{2}$.

At time $t=1$, both liquidity traders and speculators submit their orders to the MM, before the equilibrium price $P$ has been set. Liquidity traders generate a random, normally distributed demand $z$, with mean zero and variance $\sigma_{z}^{2}$. For simplicity, we assume that $z$ is independent from all other random variables. We denote the speculators' market order by $x$; thus, their profits from trading are given by $\pi=x(v-P)$. Conditional on $S$, the speculators choose their position in the risky asset to maximize their expected CARA utility

$$
E U=E[-\exp \{-\alpha \pi\} \mid S]
$$

It is well known that the above problem is equivalent to the speculators maximizing the meanvariance $(\mathrm{MV})$ function

$$
E U_{M V}=E[\pi \mid S]-\frac{1}{2} \alpha \operatorname{var}[\pi \mid S]
$$

with respect to $x$ (e.g., Huang and Litzenberger, 1988, pp. 265-266). Standard formulas for the moments of a conditional normal distribution (e.g., Greene, 1997, p. 90) imply that the unique solution of this strictly concave program is:

$$
x_{M V}=\frac{\phi S-P}{\alpha \sigma_{v}^{2}(1-\phi)},
$$

where $\phi=\frac{\sigma_{v}^{2}}{\sigma_{s}^{2}}$ is the relative precision of the speculators' private signal $S$. In Eq. (3), risk aversion induces the speculators, even if better informed and competitive, to submit cautious market orders $\left(\left|x_{M V}\right|<\infty\right)$ to the MM. 


\subsection{Market Liquidity and Price Efficiency}

The MM does not receive any information, but observes the aggregate order flow $\omega=x_{M V}+z$ from all market participants before setting the market-clearing price $P=P(\omega)$. Dealership competition and risk-neutrality then imply semi-strong market efficiency:

$$
P(\omega)=E(v \mid \omega) .
$$

We provide the resulting closed-form expression for the equilibrium price satisfying the zeroexpected profit condition of Eq. (4) in the following proposition.

Proposition 1 The unique rational expectations equilibrium price $P_{M V}$ of the model described by Eqs. (1) to (4) is

$$
P_{M V}=\lambda_{M V} \omega
$$

where

$$
\lambda_{M V}=\frac{\sigma_{v}^{2}}{\alpha \sigma_{u}^{2} \sigma_{z}^{2}}>0 .
$$

The equilibrium market liquidity (depth) in $P_{M V}$ - the inverse of the price impact $\lambda_{M V}$ of Eq. (6) — reflects the MM's attempt to be compensated for the losses he anticipates from trading with better-informed speculators, as it affects his profits from liquidity trading. Consistent with Kyle (1985), market liquidity deteriorates ( $\lambda_{M V}$ increases) the more uncertain is the risky asset's liquidation value $v$ (i.e., the greater is $\sigma_{v}^{2}$ ) since the more valuable is speculators' private information, the more aggressive is their trading activity (e.g., for any $P_{M V}, \frac{\partial\left|x_{M V}\right|}{\partial \sigma_{v}^{2}}=\frac{\left|x_{M V}\right| P_{M V}}{x_{M V} \alpha \sigma_{v}^{2}}$ ), and the more vulnerable is the MM to adverse selection. Accordingly, market liquidity improves if the speculators' private signal $S$ is less precise (i.e., the greater is $\sigma_{u}^{2}$ ) or in the presence of more intense noise trading (i.e., the greater is $\sigma_{z}^{2}$ ). Consistent with Subrahmanyam (1991), market liquidity deteriorates the less risk averse the speculators are (i.e., the lower is $\alpha$ ) for they trade

more aggressively with $S$. The following corollary summarizes these basic properties of $\lambda_{M V}$ of Eq. (6). 
Corollary 1 Equilibrium market liquidity is decreasing in $\sigma_{v}^{2}$ and increasing in $\alpha, \sigma_{u}^{2}$, and $\sigma_{z}^{2}$.

These parameters also affect the extent to which prices reveal private information in the equilibrium of Proposition 1. As in Subrahmanyam (1991), we define the informational efficiency (informativeness) of prices to be the posterior precision of the asset payoff $v$ conditional on the price $P$ (or the order flow $\omega$ ) as

$$
Q \equiv \operatorname{var}[v \mid P]^{-1}
$$

Using properties of the normal distributions and Eqs. (3), (5), and (6), it is straightforward to show that $Q_{M V}^{-1}=\sigma_{v}^{2}-\operatorname{var}\left[P_{M V}\right]$, where

$$
\operatorname{var}\left[P_{M V}\right]=\frac{\sigma_{v}^{4}}{\sigma_{u}^{2}\left(\alpha^{2} \sigma_{u}^{2} \sigma_{z}^{2}+1\right)+\sigma_{v}^{2}}
$$

We then have Corollary 2.

Corollary 2 Equilibrium price informativeness is decreasing in $\sigma_{v}^{2}, \alpha, \sigma_{u}^{2}$, and $\sigma_{z}^{2}$.

Intuitively, price efficiency $Q_{M V}$ is decreasing in the uncertainty surrounding the risky asset's payoff $v$, as in Kyle (1985). Furthermore, $Q_{M V}$ is decreasing in the quality of the speculators' private information (i.e., the greater is $\sigma_{u}^{2}$ ) and their risk aversion (i.e., the higher is $\alpha$ ) for those speculators trade less aggressively with their signal $S$, pushing $P_{M V}$ away from $v$. In Kyle (1985), noise trading does not destabilize equilibrium prices for it brings forth more trading by risk-neutral informed agents. Kyle (1985) and Subrahmanyam (1991) observe that price informativeness does instead decline in the presence of imperfectly competitive, risk-averse informed agents, for they trade less aggressively in response to a greater $\sigma_{z}^{2}$. In our setting, price informativeness declines in $\sigma_{z}^{2}$ as well, yet because more intense liquidity trading does not affect the trading activity of perfectly competitive, risk-averse speculators, hence unequivocally reduces the adverse selection risk for the MM. 


\section{A Model of Trading with Prospect Theory}

In their seminal work, Kahneman and Tversky (1979) propose Prospect Theory as a model of decision-making under uncertainty based on experimental evidence of violations of the standard Morgenstern-von Neumann utility theory. The main features of Prospect Theory are: i) a value function defined on changes in financial wealth, displaying concavity in the domain of gains (risk aversion) and convexity in the domain of losses (risk seeking), and steeper for losses than for gains (loss aversion); and ii) nonlinear and asymmetric transformations of the objective cumulative probability distribution of outcomes. This theory is supported by numerous experimental studies of human behavior in the psychology literature. ${ }^{8}$ In this section we amend the basic model of Section 2 to examine the impact of the presence of speculators with Prospect Theory-inspired preferences on equilibrium market liquidity and price informativeness.

\subsection{Prospect Theory Speculators}

We assume that, within our benchmark economy, a fraction $\mu$ of the competitive, better-informed agents makes trading decisions under Prospect Theory (PT), while the rest (MV) trades according to Eq. (3). Tversky and Kahneman (1992) propose a specific power utility function over trading gains and losses $\pi$ based on experimental evidence:

$$
U_{P T}= \begin{cases}\pi^{0.88} & \text { if } \pi \geq 0 \\ -2.25(-\pi)^{0.88} & \text { if } \pi<0 .\end{cases}
$$

This functional form, plotted in Figure 1 (dotted line), captures the main element of Prospect Theory for it is mildly concave over gains, and convex and steeper over losses. Yet, it makes the PT speculators' problem intractable to solve in our setting. Therefore, in the spirit of Barberis, Huang, and Santos (2001) and Barberis and Huang (2001), we assume that PT speculators choose

\footnotetext{
${ }^{8}$ E.g., see the surveys in Camerer (2000), Barberis, Huang, and Santos (2001), and Nofsinger (2005).
} 
the optimal trading strategy that maximizes the following piecewise expected utility:

$$
E U_{M V+P T}=E U_{M V}+E[V(\pi) \mid S]
$$

where

$$
V(\pi)=\left\{\begin{array}{cc}
0 & \text { if } \pi \geq 0 \\
\gamma \pi & \text { if } \pi<0
\end{array}\right.
$$

and $\gamma>0 .{ }^{9}$ This value function — whose realizations over the domain of $\pi$ are plotted in Figure 1 (solid line) for $\alpha=1$ and $\gamma=3$ under the assumption that $P=0$ - has the following properties: i) it reduces to MV speculators' strictly concave utility (thin line) when $\gamma=0$, ii) it leaves PT speculators as risk-averse as MV speculators over gains; iii) it is kinked at the origin (where trading gains are zero) and steeper over losses, making PT speculators loss-averse; and iv) it adds a risk-neutral term (dashed line) to PT speculators' utility when $\pi<0$, making them less risk-averse than MV speculators over losses. Thus, Eqs. (10) and (11) capture the main properties of Eq. (9) parsimoniously. It will become clearer later that our results are likely robust to (or even strengthened by) explicitly modeling PT speculators' risk-seeking behavior. ${ }^{10}$

\subsection{Prospect Theory Trading}

As in Section 2.1, sometime between $t=0$ and $t=1$, all speculators receive the same private and noisy signal of $v, S$. At time $t=1$, liquidity traders, MV speculators, and PT speculators

\footnotetext{
${ }^{9}$ For instance, Barberis, Huang, and Santos (2001) model Prospect Theory-inspired preferences in a standard consumption-based asset pricing model à la Lucas (1978) by assuming that a continuum of identical infinitely lived agents chooses a consumption level $c_{t}$ and an allocation of the risky asset $x_{t}$ to maximize $E\left[\sum_{t=0}^{\infty}\left(\rho^{t} \frac{c_{t}^{1-\gamma}}{1-\gamma}\right)+b_{t} \rho^{t+1} v\left(\pi_{t+1}\right)\right]$, where the trading gain/loss $\pi_{t+1}=x_{t}\left(R_{t+1}-R_{f, t}\right)$ while $v\left(\pi_{t+1}\right)=\pi_{t+1}$ if $\pi_{t+1} \geq 0$ and $v\left(\pi_{t+1}\right)=\lambda \pi_{t+1}($ with $\lambda>1)$ otherwise.

${ }^{10}$ For instance, more involved (unreported) analysis shows that this is the case when replacing $E U_{M V+P T}$ of Eqs. (10) and (11) with the value function $\left\{E[\pi \mid S, \pi \geq 0]-\frac{1}{2} \alpha \operatorname{var}[\pi \mid S, \pi \geq 0]\right\} \operatorname{Pr}[\pi \geq 0 \mid S]+$ $\gamma\left\{E[\pi \mid S, \pi<0]+\frac{1}{2} \beta \operatorname{var}[\pi \mid S, \pi<0]\right\} \operatorname{Pr}[\pi<0 \mid S]$, where $\beta>0$, in the spirit of Kyle, Ou-Yang, and Xiong (2006).
} 
submit their optimal market orders to the MM. MV speculators submit the demand function $x_{M V}$ of Eq. (3). PT speculators submit the demand function $x_{P T}$ maximizing $E U_{M V+P T}$ of Eq. (10). Standard formulas for the moments of a truncated normal distribution (e.g., Greene, 1997, pp. 950-952) imply that

$$
E[\pi \mid S, \pi<0]=x(\phi S-P)-x \operatorname{sgn}(x) \sqrt{\sigma_{v}^{2}(1-\phi)} \frac{\psi(\chi)}{\Phi(\operatorname{sgn}(x) \chi)},
$$

where $\operatorname{sgn}(\cdot)$ is the sign function, $\Phi(\cdot)$ and $\psi(\cdot)$ are the standard normal cdf and pdf, respectively, $\chi=\frac{P-\phi S}{\sqrt{\sigma_{v}^{2}(1-\phi)}}$, and $\Phi(\operatorname{sgn}(x) \chi)$ is the conditional cumulative probability of a trading loss. Let $\operatorname{sgn}(x)=1$. It then ensues that Eq. (12) becomes

$$
E[\pi \mid S, \pi<0]=x(\phi S-P)-x \sqrt{\sigma_{v}^{2}(1-\phi)} \frac{\psi(\chi)}{\Phi(\chi)}
$$

and that

$$
E U_{M V+P T}=E U_{M V}+\gamma E[\pi \mid S, \pi<0] \Phi(\chi) .
$$

Besides being computationally convenient, the above assumption transforms PT speculators' probabilities nonlinearly and asymmetrically (in the spirit of Kahneman and Tversky, 1979; Tversky and Kahneman, 1992; Barberis and Huang, 2008), by overweighting low cumulative probabilities of trading losses $(\Phi(\chi)>\Phi(\operatorname{sgn}(x) \chi)$ when $\chi>\operatorname{sgn}(x) \chi)$. This property captures economic agents' tendency to be disproportionately afraid of the low odds of losing when choosing gambles with high odds of winning (e.g., Jullien and Salanié, 2000). The resulting subjective (i.e., transformed) probabilities of the objective ones — a model device capturing decision weights rather than "incorrect" beliefs - play an important role in our analysis. Nonetheless, their omission, while complicating such analysis considerably, does not change the qualitative nature of our main results and intuition. ${ }^{11}$

\footnotetext{
${ }^{11}$ In addition, our analysis yields qualitatively similar basic results for liquidity and price efficiency if we let $\operatorname{sgn}(x)=-1$.
} 
Substituting Eq. (2) into Eq. (14) and differentiating with respect to $x$, we then have

$$
x_{P T}=[1+\gamma \Phi(\chi)] x_{M V}-\frac{\gamma}{\alpha \sqrt{\sigma_{v}^{2}(1-\phi)}} \psi(\chi) .
$$

Intuitively, the stylized Prospect Theory preferences of Eq. (10) (i.e., $\gamma>0$ in $x_{P T}$ ) have two effects of the opposite sign on the optimal trading activity of PT speculators as compared to that of MV speculators $\left(x_{M V}\right)$. For any given signal $S$ and price $P$, lower risk aversion in losses (which we label risk seeking) induces PT speculators to more aggressive trading, the more so the greater is $\Phi(\chi)$, the conditional subjective cumulative probability of a trading loss; loss aversion instead induces PT speculators to more cautious trading in order to minimize the expected loss of Eq. (13), the more so the greater is $\Phi^{\prime}(\chi)=\psi(\chi)$, the conditional marginal probability of a trading loss. To gain further insight on Eq. (15), we construct a simple numerical example by setting $\sigma_{v}^{2}=\sigma_{u}^{2}=\sigma_{z}^{2}=1, \alpha=1$, and $\gamma=3$. We then plot PT and MV speculators' resulting demand schedules $x_{P T}$ (solid line) and $x_{M V}$ (thin line), as well as $(1+\gamma) x_{M V}$ (dashed line) in Figure 2 over the domain of $P$ for a private signal $S=0$. When prices are low $(P \ll 0)$, a loss is subjectively and objectively unlikely $(\Phi(\chi)=\Phi(\operatorname{sgn}(x) \chi) \approx 0$ and $\psi(\chi) \approx 0)$ and PT speculators act as MV speculators $\left(x_{P T} \approx x_{M V}\right)$. When prices are high $(P \gg 0)$, a loss is subjectively, although not objectively, likely $(\Phi(\chi) \approx 1$ although $\Phi(\operatorname{sgn}(x) \chi) \approx 0$, while $\psi(\chi) \approx 0)$ and risk seeking dominates PT speculators' trading strategy $\left(x_{P T} \approx(1+\gamma) x_{M V}\right)$. Everywhere else, PT speculators' optimal trading strategy depends upon the trade-off between loss aversion and risk seeking. For instance, when $P=-0.5$, loss aversion leads PT speculators to more cautious trading than MV speculators' $\left(x_{M V}=1\right.$ but $\left.x_{P T}=0.40\right)$ because the marginal probability of a loss is relatively high $(\psi(P=-0.5)>\Phi(P=-0.5))$; when instead $P=0.5$, risk-seeking leads PT speculators to more aggressive trading than MV speculators' ( $x_{M V}=-1$ but $\left.x_{P T}=-4.60\right)$ because the subjective cumulative probability of a loss is relatively high $(\Phi(P=0.5)>\psi(P=0.5))$. 


\subsection{Market Liquidity and Price Efficiency}

As in Section 2.2, the MM sets the equilibrium market price according to Eq. (4), after observing the aggregate order flow $\omega=\mu x_{P T}+(1-\mu) x_{M V}+z$ from all market participants. Unfortunately, the expression for $x_{P T}$ in Eq. (15) makes $\omega$ a highly nonlinear function of the normally distributed private signal $S$, thus the MM's problem analytically intractable. There are several approaches in the literature for approximating nonlinear rational expectation models to the first order of accuracy. ${ }^{12}$ We look for a first-order accurate solution to our model by employing a first-order approximation of $x_{P T}$ around the private signal's unconditional mean $E[S]=0$.

Lemma 1 The first-order representation of PT speculators' optimal trading strategy of Eq. (15) is given by:

$$
x_{P T} \approx[1+\gamma \Phi(\bar{\chi})] x_{M V}-\frac{\gamma}{\alpha \sqrt{\sigma_{v}^{2}(1-\phi)}} \psi(\bar{\chi})
$$

where $\bar{\chi} \equiv E[\chi]=\frac{P}{\sqrt{\sigma_{v}^{2}(1-\phi)}}$.

This representation of $x_{P T}$ turns out to be reasonably accurate. For instance, it is virtually indistinguishable from the true $x_{P T}$ of Eq. (15) when evaluated locally (e.g., the thin gray line in Figure 2 when $S=0$ ). Eq. (16) also indicates that, in a first-order sense, risk seeking dominates loss aversion in PT speculators' market orders as a source of adverse selection risk for the MM, since the approximate loss aversion portion of $x_{P T}$ is deterministic. Proposition 2 accomplishes the task of solving for the semi-strong efficient market-clearing price of this economy given Eq. (16).

Proposition 2 The first-order rational expectations equilibrium of the amended economy described by Eqs. (3), (4), and (16) when $\mu>0$ is uniquely characterized by

$$
P_{P T}=\lambda_{P T}\left[\omega+\frac{\mu \gamma}{\alpha \sqrt{\sigma_{v}^{2}(1-\phi)}} \psi(\bar{\chi})\right]
$$

\footnotetext{
${ }^{12}$ E.g., see Blanchard and Kahn (1980), Klein (2000), and Sims (2000), as well as the discussion in Lombardo and Sutherland (2007).
} 
where

$$
\lambda_{P T}=[1+\mu \gamma \Phi(\bar{\chi})] \lambda_{M V}>\lambda_{M V}
$$

A risky asset's equilibrium market liquidity in the presence of PT speculators has two noteworthy properties, novel to the literature. First, the asset's equilibrium market depth is lower than in the absence of such speculators. Intuitively, PT speculators' trading activity — stemming from the optimal resolution of a trade-off between loss aversion and risk seeking in Eq. (14) — is more aggressive than that of MV speculators, thus making the MM more vulnerable to adverse selection and the price impact of aggregate order flow higher. Second, the asset's equilibrium market depth is state-dependent. As suggested by Lemma 1, the extent to which PT speculators' optimal market orders depart from those of MV speculators in equilibrium — hence, the MM's perceived adverse selection risk — depends mainly on the expected subjective cumulative probability of a loss $(\Phi(\bar{\chi}))$, a function of the realized order flow $\omega$ (i.e., of the realizations of the private signal $S$ and the amount of noise trading $z$ ) via $P_{P T}$. Foster and Viswanathan (1993) show that representing agents' beliefs with nonnormal elliptically contoured distributions is sufficient to make market liquidity state-dependent in a model of market-making à la Kyle (1985) with endogenous information acquisition. In our model, state-dependent market liquidity ensues from state-dependent preferences even when all random variables are normally distributed.

As Proposition 2 makes clear, the expression for $\lambda_{P T}$ and its relationship with the model's state variables $S$ and $z$ are analytically complicated since $P_{P T}$ is a fixed point of Eq. (17). We therefore examine the state dependency of equilibrium market depth via a numerical example. Specifically, in the upper panel of Figure 3 we plot (by virtue of numerical integration) the average price impact $\lambda_{P T}$ and PT speculators' optimal trading activity with respect to all possible noise trading shocks $z$ over the domain of $S$ (i.e., $E\left[\lambda_{P T} \mid S\right]$ and $E\left[x_{P T} \mid S\right]$, respectively) when $\gamma=3$ and $\mu=0.1$, as well as their benchmark counterparts when $\mu=0$ (i.e., $\lambda_{M V}$ and $E\left[x_{M V} \mid S\right]$ ), in the economy of Figure 2. As previously discussed, the presence of PT speculators always worsens 
a risky asset's equilibrium market liquidity: $E\left[\lambda_{P T} \mid S\right]$ (solid line) $>\lambda_{M V}$ (dotted line).

In addition, market depth becomes countercyclical in the presence of PT speculators: $E\left[\lambda_{P T} \mid S\right]$ is increasing in $S$, i.e., equilibrium price impact is greater during "good" times $(S>0)$ than during "bad" times $(S<0)$. The intuition for this result is that in equilibrium, the subjective cumulative probability of a trading loss $(E[\Phi(\bar{\chi}) \mid S]$, solid gray line in the lower panel of Figure 3, controlling the extent of PT speculators' risk seeking in trading) is increasing in $S$ (i.e., procyclical) — while the marginal probability of a trading loss $E[\psi(\bar{\chi}) \mid S]$ (thin gray line in the lower panel of Figure 3, controlling the extent of PT speculators' loss aversion in trading) is decreasing in $|S|$ - since so is the market-clearing price $P_{P T}$. The resulting increasing risk seeking and declining loss aversion portions of Eq. (16) induce PT speculators to trade more aggressively ( $E\left[x_{P T} \mid S\right]$, thin line, as compared to $E\left[x_{M V} \mid S\right]$, thin dotted line) and thus make the MM more vulnerable to adverse selection. Similar inference ensues from plotting $E\left[\lambda_{P T} \mid S\right]$ with respect to $P_{P T}$. As such, the equilibrium market liquidity of a risky asset traded by PT speculators is inversely related to its (possibly unobservable) fundamental performance, hence to its closest (observable) proxy, the asset's simultaneously determined price performance.

We next assess further properties of the equilibrium of Proposition 2 by employing the Law of Iterated Expectations on $E\left[P_{P T}\right]$ to approximate the first moment of $\lambda_{P T}\left(E\left[\lambda_{P T}\right]\right)$ and the second moment of $P_{P T}\left(\operatorname{var}\left[P_{P T}\right]\right)$ as follows:

Corollary 3 Eqs. (17) and (18) imply that

$$
E\left[\lambda_{P T}\right] \approx\left(1+\frac{1}{2} \mu \gamma\right) \lambda_{M V}
$$

and

$$
\operatorname{var}\left[P_{P T}\right] \approx \frac{\left(1+\frac{1}{2} \mu \gamma\right)^{2} \sigma_{v}^{4}}{\left(1+\frac{1}{2} \mu \gamma\right)^{2}\left(\sigma_{v}^{2}+\sigma_{u}^{2}\right)+\alpha^{2} \sigma_{u}^{4} \sigma_{z}^{2}}>\operatorname{var}\left[P_{M V}\right]
$$

Unreported numerical integration shows that these expressions are approximately equal to the true mean of $\lambda_{P T}$ of Eq. (18) and variance of $P_{P T}$ of Eq. (17). According to Eq. (19), both 
a greater presence of PT speculators among informed traders (i.e., higher $\mu$ ) and a more severe kink in their expected utility function of Eq. (14) (i.e., higher $\gamma$ ) deteriorate a risky asset's average equilibrium market liquidity for either of them worsens the MM's perceived adverse selection risk. Consistently, $E\left[\lambda_{P T}\right]$ is more sensitive to fundamental uncertainty $\left(\sigma_{v}^{2}\right)$, agents' risk aversion $(\alpha)$, the quality of their private information $\left(\sigma_{u}^{2}\right)$, and the intensity of liquidity trading $\left(\sigma_{z}^{2}\right)$ than in the absence of PT speculators (Corollary 1), since so is the latter's more aggressive trading activity.

Lastly, Eq. (4) and Corollary 3 imply that

$$
Q_{P T}^{-1}=\sigma_{v}^{2}-\operatorname{var}\left[P_{P T}\right]<Q_{M V}^{-1}
$$

a closed-form expression from which the following remark ensues.

Remark 1 The presence of PT speculators improves equilibrium price informativeness, the more so the greater is their fraction of the total population of speculators (higher $\mu$ ) and/or the more pronounced are their PT preferences (higher $\gamma$ ). Its comparative statics are otherwise the same as in Corollary 2.

Barberis, Huang, and Santos (2001) and Barberis and Huang (2001) study the asset pricing implications of loss aversion at the kink of a stylized, state-dependent specification of agents' Prospect Theory value function — but not of its curvature over gains and losses and its probability weighting component — in an otherwise standard consumption-based asset pricing model à la Lucas (1978). In such a setting, they show that state-dependent loss aversion makes those agents' risk aversion time-varying, eventually leading to excess equilibrium price volatility over that of the underlying dividends. ${ }^{13}$ In our model of trading, the risk seeking portion of speculative PT trading dominates the loss aversion one (e.g., see Figure 2). This makes PT speculators' trading

\footnotetext{
${ }^{13}$ Consistently, in unreported analysis we show that introducing loss aversion in the preferences of an otherwise risk-neutral monopolist speculator in the basic setting of Kyle (1985) leads to more cautious informed trading, greater market liquidity, and worse price informativeness in equilibrium.
} 
activity more aggressive than MV speculators', and the aggregate order flow more informative about the risky asset's liquidation value $v\left(\operatorname{var}\left[P_{P T}\right]>\operatorname{var}\left[P_{M V}\right]\right)$, thus not only worsening equilibrium market liquidity but also improving equilibrium price efficiency. ${ }^{14}$

\section{Extension: A Public Signal}

An important characteristic of most financial markets is the frequent release of news about the fundamentals of the traded securities to all market participants. There is a vast literature showing that such release affects both the dynamics of asset prices and the liquidity of their trading venues. ${ }^{15}$ In this section, we are interested in the sensitivity of the impact of such releases on adverse selection-based market liquidity and price informativeness to the presence of PT speculators. To that purpose, we extend the model of Sections 2 and 3 by providing each agent with an additional, common source of information about the risky asset before trading takes place.

\subsection{The Benchmark Model}

We consider a market that is identical to that of Section 2. We then assume that before trading takes place, sometime between $t=0$ and $t=1$, both the speculators and the MM observe a public and noisy signal of the risky asset's payoff $v, S_{p}=v+e$. We can think of $S_{p}$ as

\footnotetext{
${ }^{14}$ Note, however, that equilibrium price informativeness is not state-dependent, for any realization of $S$ equally affects both the conditional variance of the aggregate order flow and its conditonal covariance with the risky asset's final payoff $v$, thus leaving both $\operatorname{var}\left[P_{P T} \mid S\right]$ and $E\left[Q_{P T} \mid S\right]$ unchanged in equilibrium (see Eq. (A-15) in the Appendix).

${ }^{15}$ An incomplete list includes Admati and Pfleiderer (1988), Foster and Viswanathan (1990, 1993), McKelvey and Page (1990), Diamond and Verrecchia (1991), Berry and Howe (1994), Kim and Verrecchia (1994, 1997), Andersen and Bollerslev (1998), Fleming and Remolona (1999), Evans and Lyons (2003), Brandt and Kavajecz (2004), Green (2004), Boyd, Hu, and Jagannathan (2005), and Pasquariello and Vega (2007).
} 
any public announcement (e.g., earnings or macroeconomic news) released simultaneously to all market participants. The signal noise $e$ is normally distributed with mean zero and variance $\sigma_{e}^{2}$ and independent from any other random variable. It then ensues that $\operatorname{var}\left[S_{p}\right] \equiv \sigma_{p}^{2}=\sigma_{v}^{2}+\sigma_{e}^{2}$, $\operatorname{cov}\left[v, S_{p}\right]=\sigma_{v}^{2}$, and $\operatorname{cov}\left[S, S_{p}\right]=\sigma_{v}^{2}$.

The availability of $S_{p}$ affects the level and improves the precision of the information of all market participants prior to trading at time $t=1$, with respect to the economy of Section 2.1. The MM's revised priors about the distribution of $v$ before trading occurs are now given by $E\left[v \mid S_{p}\right]=\phi_{p} S_{p}$ and $\sigma_{v}^{2 *} \equiv \operatorname{var}\left[v \mid S_{p}\right]=\sigma_{v}^{2}\left(1-\phi_{p}\right)$, where $\phi_{p}=\frac{\sigma_{v}^{2}}{\sigma_{p}^{2}}$ measures the relative precision of the public signal $S_{p}$. Accordingly, the speculators' revised priors about the distribution of $v$ before trading occurs but after observing their private signal $S$ are $E\left[v \mid S, S_{p}\right]=\left(1-\phi^{*}\right) \phi_{p} S_{p}+$ $\phi^{*} S$ and $\operatorname{var}\left[v \mid S, S_{p}\right]=\sigma_{v}^{2 *}\left(1-\phi^{*}\right)$, where $\phi^{*}=\frac{\sigma_{v}^{2 *}}{\sigma_{s}^{2 *}}$ measures the relative precision of the private signal $S$ conditional upon observing $S_{p}$ and $\sigma_{s}^{2 *} \equiv \operatorname{var}\left[S \mid S_{p}\right]=\sigma_{v}^{2 *}+\sigma_{u}^{2}$.

Informed traders in the benchmark economy are made solely of MV speculators. The above assumptions straightforwardly imply that their optimal trading strategy after a public signal $S_{p}$ is released is

$$
x_{M V}^{*}=\frac{\left(1-\phi^{*}\right) \phi_{p} S_{p}+\phi^{*} S-P}{\alpha \sigma_{v}^{2 *}\left(1-\phi^{*}\right)},
$$

while the MM's market-clearing price is

$$
P\left(\omega, S_{p}\right)=E\left(v \mid \omega, S_{p}\right)
$$

Proposition 3 describes the equilibrium of this amended economy.

Proposition 3 The unique rational expectations equilibrium price $P_{M V}^{*}$ of the model described by Eqs. (22) and (23) is

$$
P_{M V}^{*}=\phi_{p} S_{p}+\lambda_{M V}^{*} \omega
$$

where

$$
\lambda_{M V}^{*}=\left(1-\phi_{p}\right) \lambda_{M V}<\lambda_{M V}
$$


Consistent with Foster and Viswanathan (1993) and Pasquariello and Vega (2007), the release of a public signal improves market liquidity in subsequent trading. The availability of $S_{p}$ has two effects of opposite sign on the MM's perceived adverse selection risk. First, it makes speculators' private information less valuable, hence the MM's adverse selection risk less severe for it provides him with a trade-free source of information about the traded asset's final payoff $v$. Second, it makes the trading activity of risk-averse MV speculators in the risky asset more aggressive for it reduces the conditional variance of $v\left(\sigma_{v}^{2 *}<\sigma_{v}^{2}\right)$. This is illustrated in Figure 4, which plots MV speculators' optimal market orders $x_{M V}$ of Eq. (3) (dotted line) and $x_{M V}^{*}$ of Eq. (solid line) over the domain of $P$ for a private signal $S=0$ and a public signal $S_{p}=0$ in the economy of Figure 2. As clear from Figure 4, the availability of $S_{p}$ causes MV speculators' optimal trading strategy to tilt up at the origin (since $S_{p}=0$ ). In equilibrium, the first effect dominates the second and market depth improves. Accordingly, the percentage improvement in liquidity, defined as

$$
\Delta \lambda_{M V}^{*} \equiv \frac{\lambda_{M V}^{*}}{\lambda_{M V}}-1=-\phi_{p}<0
$$

is increasing in $\phi_{p}$, the relative precision of $S_{p}$, i.e., in its value and quality, as follows.

Remark 2 The percentage decrease in the order flow's price impact when $S_{p}$ is available is increasing in $\sigma_{v}^{2}$ and decreasing in $\sigma_{e}^{2}$.

The availability of a public signal also improves equilibrium price informativeness. In particular, Eq. (7) and the above distributional assumptions imply that $\left(Q_{M V}^{*}\right)^{-1}=\sigma_{v}^{2 *}-\operatorname{var}\left[P_{M V}^{*} \mid S_{p}\right]<$ $Q_{M V}^{-1}$, where

$$
\operatorname{var}\left[P_{M V}^{*} \mid S_{p}\right]=\frac{\sigma_{v}^{4 *}}{\sigma_{u}^{2}\left(\alpha^{2} \sigma_{u}^{2} \sigma_{z}^{2}+1\right)+\sigma_{v}^{2 *}}<\operatorname{var}\left[P_{M V}\right]
$$

since $\sigma_{v}^{2 *}<\sigma_{v}^{2}$. Thus, a trade-free source of information always ameliorates price efficiency despite the order flow becoming less informative about $v$ - the more so the greater is the quality of the public signal (i.e., the lower is $\sigma_{e}^{2}$ ). We then have Corollary 4 . 
Corollary 4 Equilibrium price informativeness when $S_{p}$ is available is decreasing in $\sigma_{e}^{2}$. Its comparative statics are otherwise the same as in Corollary 2.

\subsection{Prospect Theory Trading}

We extend the model of Section 4.1 to allow a portion $\mu$ of the mass of speculators to be characterized by the Prospect Theory-inspired preferences of Eqs. (10) and (11). Consistent with Section 3.2, when a public signal $S_{p}$ is available PT speculators choose the demand schedule $x$ maximizing

$$
E U_{M V+P T}^{*}=E\left[\pi \mid S, S_{p}\right]-\frac{1}{2} \alpha \operatorname{var}\left[\pi \mid S, S_{p}\right]+\gamma E\left[\pi \mid S, S_{p}, \pi<0\right] \Phi\left(\chi^{*}\right),
$$

where, because of the properties of truncated conditional normal distributions and $\operatorname{sign}(x)=1$,

$$
E\left[\pi \mid S, S_{p}, \pi<0\right]=x\left[\left(1-\phi^{*}\right) \phi_{p} S_{p}+\phi^{*} S-P\right]-x \sqrt{\sigma_{v}^{2 *}\left(1-\phi^{*}\right)} \frac{\psi\left(\chi^{*}\right)}{\Phi\left(\chi^{*}\right)}
$$

and $\chi^{*}=\frac{P-\left(1-\phi^{*}\right) \phi_{p} S_{p}-\phi^{*} S}{\sqrt{\sigma_{v}^{2 *}\left(1-\phi^{*}\right)}}$. Differentiating the resulting expression for $E U_{M V+P T}^{*}$ with respect to $x$ gives

$$
x_{P T}^{*}=\left[1+\gamma \Phi\left(\chi^{*}\right)\right] x_{M V}^{*}-\frac{\gamma}{\alpha \sqrt{\sigma_{v}^{2 *}\left(1-\phi^{*}\right)}} \psi\left(\chi^{*}\right) .
$$

The optimal trading strategy of PT speculators in the presence of a public signal is similar to $x_{P T}$ of Eq. (15). As discussed in Section 3.2, their market orders are driven by three effects: First, risk aversion leads to cautious trading, as for MV speculators, the more so the greater is the uncertainty surrounding the asset's final payoff $v$; second, risk seeking leads to more aggressive trading, the more so the greater is the conditional subjective cumulative probability of a loss given $S$ and $S_{p}, \Phi\left(\chi^{*}\right)$; third, loss aversion leads to more cautious trading, the more so the greater is the conditional marginal probability of a loss given $S_{p}, \Phi^{\prime}\left(\chi^{*}\right)=\psi\left(\chi^{*}\right)$. Yet, the extent to which these forces manifest and interact in Eq. (30) is now affected by the availability of $S_{p}$ in a complex fashion, since the latter not only reduces the conditional variance of $v\left(\sigma_{v}^{2 *}\left(1-\phi^{*}\right)<\sigma_{v}^{2}(1-\phi)\right)$ 
but also affects both $\Phi\left(\chi^{*}\right)$ and $\psi\left(\chi^{*}\right)$. For instance, Figure 4 plots PT speculators' optimal market orders $x_{P T}$ of Eq. (15) (dashed gray line) and $x_{P T}^{*}$ of Eq. (30) (solid line) over the domain of $P$ for a private signal $S=0$ and a public signal $S_{p}=0$ in the economy of Figure 2 . In this example, the availability of $S_{p}$ makes PT speculators' optimal trading strategy generally more aggressive except in the region of $P$ by the origin, where loss aversion (so trading caution) is most relevant.

As in Section 3.2, we look for a first-order accurate equilibrium of this amended economy by employing a first-order approximation of $x_{P T}^{*}$ around the private signal's conditional mean given $S_{p}, E\left[S \mid S_{p}\right]=\phi_{p} S_{p}$. Using arguments similar to those in the proof to Lemma 1, we get

$$
x_{P T}^{*} \approx\left[1+\gamma \Phi\left(\bar{\chi}^{*}\right)\right] x_{M V}^{*}-\frac{\gamma}{\alpha \sqrt{\sigma_{v}^{2 *}\left(1-\phi^{*}\right)}} \psi\left(\bar{\chi}^{*}\right),
$$

where $\bar{\chi}^{*}=\frac{P-\phi_{p} S_{p}}{\sqrt{\sigma_{v}^{2 *}\left(1-\phi^{*}\right)}}$. Again, the first-order representation of $x_{P T}^{*}$ in Eq. (31) is reasonably accurate. E.g., the plot of this approximation over the domain of $P$ in Figure 4 (thin gray line) is virtually indistinguishable from that of $x_{P T}^{*}$ of Eq. (30). The ensuing equilibrium is in Proposition 4.

Proposition 4 The first-order rational expectations equilibrium of the amended economy described by Eqs. (22), (23), and (31) when $\mu>0$ is uniquely characterized by

$$
P_{P T}^{*}=\phi_{p} S_{p}+\lambda_{P T}^{*}\left[\omega+\frac{\mu \gamma}{\alpha \sqrt{\sigma_{v}^{2 *}\left(1-\phi^{*}\right)}} \psi\left(\bar{\chi}^{*}\right)\right]
$$

where

$$
\lambda_{P T}^{*}=\left[1+\mu \gamma \Phi\left(\bar{\chi}^{*}\right)\right] \lambda_{M V}^{*}<\lambda_{P T}
$$

Proposition 4 generates novel predictions for the impact of the release of public news on market liquidity and price efficiency. According to Eq. (33), the availability of public information improves market liquidity regardless of whether a fraction of informed trading in the aggregate order flow comes from PT speculators. In particular, Proposition 4 indicates that a public signal 
$S_{p}$ of $v$ alleviates the MM's perceived adverse selection risk more than the greater aggressiveness of both PT and MV speculators' optimal trading strategies (e.g., as displayed in Figure 4) in response to $S_{p}$ may aggravate it. As in Eq. (26), we measure the extent of such improvement in market depth when $\mu>0$ as

$$
\Delta \lambda_{P T}^{*}=R\left(\bar{\chi}^{*}, \bar{\chi}\right)\left(1-\phi_{p}\right)-1<0
$$

where $R\left(\bar{\chi}^{*}, \bar{\chi}\right)=\frac{1+\mu \gamma \Phi\left(\bar{\chi}^{*}\right)}{1+\mu \gamma \Phi(\bar{\chi})}$. We learn further properties of this equilibrium by applying the Law of Iterated Expectations to $E\left[P_{P T}^{*}\right]$ in the following corollary.

Corollary 5 Eqs. (32), (33), and (34) imply that

$$
\begin{aligned}
E\left[\lambda_{P T}^{*} \mid S_{p}\right] & \approx\left(1+\frac{1}{2} \mu \gamma\right) \lambda_{M V}^{*}, \\
\operatorname{var}\left[P_{P T}^{*} \mid S_{p}\right] & \approx \frac{\left(1+\frac{1}{2} \mu \gamma\right)^{2}\left(\sigma_{v}^{2 *}\right)^{2}}{\left(1+\frac{1}{2} \mu \gamma\right)^{2}\left(\sigma_{v}^{2 *}+\sigma_{u}^{2}\right)+\alpha^{2} \sigma_{u}^{4} \sigma_{z}^{2}},
\end{aligned}
$$

where $\operatorname{var}\left[P_{M V}^{*} \mid S_{p}\right]<\operatorname{var}\left[P_{P T}^{*} \mid S_{p}\right]<\operatorname{var}\left[P_{P T}\right]$, and

$$
E\left[\Delta \lambda_{P T}^{*} \mid S_{p}\right] \approx E\left[R\left(\bar{\chi}^{*}, \bar{\chi}\right) \mid S_{p}\right]\left(1-\phi_{p}\right)-1,
$$

where $E\left[R\left(\bar{\chi}^{*}, \bar{\chi}\right) \mid S_{p}\right]=\frac{1+\frac{1}{2} \mu \gamma}{1+\mu \gamma \Phi\left(\frac{\phi_{p} S_{p}}{\sqrt{\sigma_{v}^{2}(1-\phi)}}\right)}$.

Consistent with the above arguments (as in Section 4.1), the availability of a trade-free source of information about $v$ improves price efficiency more than the less volatile (i.e., informative) aggregate order flow in equilibrium may deteriorate it. Indeed, Eqs. (7) and (36) imply that $\left(Q_{P T}^{*}\right)^{-1}=\sigma_{v}^{2 *}-\operatorname{var}\left[P_{P T}^{*} \mid S_{p}\right]<Q_{P T}^{-1}$, as well as that $\left(Q_{P T}^{*}\right)^{-1}<\left(Q_{M V}^{*}\right)^{-1}$, since $\sigma_{v}^{2 *}<\sigma_{v}^{2}$. Intuitively, the availability of a public signal has a greater impact on price efficiency when, ceteris paribus for $S_{p}$, speculators as a whole trade less cautiously. Hence, price informativeness is increasing in the fraction of the total population of informed traders made of PT speculators $(\mu)$ and in the kink in their PT preferences $(\gamma)$, since both increase the informativeness of the aggregate order flow. 
Corollary 6 Equilibrium price informativeness when $S_{p}$ is available and $\mu>0$ is increasing in $\mu$ and $\gamma$. Its comparative statics are otherwise the same as in Corollary 4.

As interestingly, Proposition 4 and Corollary 5 further indicate that when a public signal $S_{p}$ is available in an economy characterized by the presence of PT speculators, equilibrium market depth and price efficiency are dependent upon realizations of both the private signal $S$ and the public signal (as well as of noise trading $z$ ). Such dependency is analytically complicated, thus best illustrated via a numerical example. Specifically, we assume that $\sigma_{e}^{2}=1$ and consider the release of neutral $(N)$, negative $(L)$, or positive $(H)$ public news - either $S_{p}^{N}=E\left[S_{p}\right]=0$, $S_{p}^{L}=E\left[S_{p}\right]-2 \sigma_{p}=-2.83$, or $S_{p}^{H}=E\left[S_{p}\right]+2 \sigma_{p}=2.83$ - in the economy of Figure 2 . We then use numerical integration to plot, in the left panel of Figure 5, the ensuing average equilibrium price impact $\lambda_{P T}^{*}$ with respect to all possible shocks $z$ over the domain of $S$ when $\gamma=3$ and $\mu=0.1-E\left[\lambda_{P T}^{*} \mid S, S_{p}^{N}\right]$ (solid line), $E\left[\lambda_{P T}^{*} \mid S, S_{p}^{L}\right]$ (dashed gray line), and $E\left[\lambda_{P T}^{*} \mid S, S_{p}^{H}\right]$ (thin line) - as well as their benchmark counterpart when $\mu=0-\lambda_{M V}^{*}$ of Eq. (25) (dotted line). We also plot, in the center panel of Figure 5, the corresponding average percentage improvement in market depth conditional upon $S$ and $S_{p}$, computed according to Eq. (34) $-E\left[\Delta \lambda_{P T}^{*} \mid S, S_{p}^{N}\right]$ (solid line), $E\left[\Delta \lambda_{P T}^{*} \mid S, S_{p}^{L}\right]$ (dashed gray line), and $E\left[\Delta \lambda_{P T}^{*} \mid S, S_{p}^{H}\right]$ (thin line) — and Eq. (26) $-E\left[\Delta \lambda_{M V}^{*} \mid S\right]$ (dotted line).

Figure 5 suggests that ceteris paribus for the private signal $S$, the better is the public news released (i.e., the higher is $S_{p}$ ) the lower is $\lambda_{P T}^{*}$ and the greater is the improvement in market liquidity stemming from its release. ${ }^{16}$ The intuition for this result is that ceteris paribus for $S$, the higher is $S_{p}$ the lower is the expected subjective cumulative loss probability $\left(E\left[\Phi\left(\bar{\chi}^{*}\right) \mid S, S_{p}\right]\right)$ relative to the case in which $S_{p}$ is unavailable $\left(E\left[\Phi(\bar{\chi}) \mid S, S_{p}\right]\right)$, hence the less aggressive is PT speculators' trading activity and the lower is the MM's perceived adverse selection risk. This effect is clearly displayed in Eq. (37), where the ratio $E\left[R\left(\bar{\chi}^{*}, \bar{\chi}\right) \mid S_{p}\right]$ is decreasing in $S_{p}$ such that

\footnotetext{
${ }^{16}$ Note that this statement is consistent with $E\left[\lambda_{P T}^{*} \mid S_{p}\right]$ of Eq. (35) being independent of $S_{p}$ since the ensuing conditional distribution of $S$ shifts accordingly.
} 
$E\left[\Delta \lambda_{P T}^{*} \mid S_{p}\right]<\Delta \lambda_{M V}^{*}$ when $S_{p}>0$ and $E\left[\Delta \lambda_{P T}^{*} \mid S_{p}\right]>\Delta \lambda_{M V}^{*}$ when $S_{p}<0$. Figure 5 further indicates that ceteris paribus for $S_{p}$, the ensuing improvement in market liquidity is procyclical as well (i.e., the absolute value of $E\left[\Delta \lambda_{P T}^{*} \mid S, S_{p}\right]$ is increasing in $S$ ) since so is the extent to which PT speculators' trading activity becomes less aggressive relative to the case in which $S_{p}$ is unavailable. Accordingly, the comparative statics of $E\left[\Delta \lambda_{P T}^{*} \mid S_{p}\right]$ are also state-dependent.

Remark 3 The expected percentage improvement in market depth due to $S_{p}$ is increasing in $\sigma_{v}^{2}$ and decreasing in $\sigma_{e}^{2}$. When $S_{p}>0$, such percentage improvement is increasing in $\mu$ and $\gamma$, and decreasing in $\sigma_{u}^{2}$. When $S_{p}<0$, such percentage improvement is decreasing in $\mu$ and $\gamma$, and increasing in $\sigma_{u}^{2}$.

The effect of either the fundamental uncertainty surrounding the risky asset's final payoff $v$ $\left(\sigma_{v}^{2}\right)$ or the quality of the public signal $\left(\sigma_{v}^{2}\right)$ on $E\left[\Delta \lambda_{P T}^{*} \mid S_{p}\right]$ is intuitive: For any $S_{p}$, the more valuable or precise is public information about $v$, the more it ameliorates the MM's adverse selection problem. This effect is either magnified or attenuated by the impact of the actual realization of $S_{p}$ on the expected relative subjective loss probability (i.e., the ratio $\left.E\left[R\left(\bar{\chi}^{*}, \bar{\chi}\right) \mid S_{p}\right]\right)$ when considering the sensitivity of $E\left[\Delta \lambda_{P T}^{*} \mid S_{p}\right]$ to $\mu, \gamma$, and $\sigma_{u}^{2}$. When good public news is released $\left(S_{p}>0\right)$, the greater is the fraction of PT speculators in the economy $(\mu)$ and the extent of their Prospect Theory-inspired preferences $(\gamma)$, the greater is the percentage improvement in market liquidity induced by $S_{p}$ : The public signal reduces the expected subjective cumulative loss probability relative to when $S_{p}$ is unavailable, thus attenuating PT speculators' trading aggressiveness. Vice versa, when bad public news is released $\left(S_{p}<0\right)$, the expected subjective cumulative loss probability relative to when $S_{p}$ is unavailable increases, thus inducing PT speculators to less caution in their trading activity. Similarly, the improvement in market liquidity following the release of a good (bad) public signal is smaller (greater) when the private signal $S$ is of poorer quality, i.e., when $\sigma_{u}^{2}$ is higher: The latter unequivocally reduces the MM's adverse selection risk, thus attenuating (magnifying) his concern for PT speculators' lower (greater) trading aggressiveness 
in the order flow. ${ }^{17}$

Lastly, ceteris paribus for $S$, price efficiency too becomes procyclical in the presence of PT speculators when a public signal is released. This is shown in the right panel of Figure 5, where we plot the average price informativeness conditional upon $S$ and $S_{p}$ computed according to Eqs. (4) and (36): $E\left[Q_{P T}^{*} \mid S, S_{p}^{N}\right]$ (solid line), $E\left[Q_{P T}^{*} \mid S, S_{p}^{L}\right]$ (dashed gray line), and $E\left[Q_{P T}^{*} \mid S, S_{p}^{H}\right]$ (thin line). $E\left[Q_{P T}^{*} \mid S, S_{p}\right]$ is increasing in both $S$ and $S_{p}$, i.e., price efficiency is greater during good times $\left(S>0\right.$ or $\left.S_{p}>0\right)$ than during bad times $\left(S<0\right.$ or $\left.S_{p}>0\right)$. Intuitively, ceteris paribus for $S\left(S_{p}\right)$, a higher public (private) signal $S_{p}(S)$ increases PT speculators' expected subjective cumulative loss probability $\left(E\left[\Phi\left(\bar{\chi}^{*}\right) \mid S, S_{p}\right]\right)$ - hence enhancing their risk-seeking behavior - and decreases their expected marginal loss probability $\left(E\left[\psi\left(\bar{\chi}^{*}\right) \mid S, S_{p}\right]\right)$ — hence attenuating their loss-averse behavior — via $P_{P T}^{*}$. In equilibrium, these two effects lead PT speculators to more aggressive trading, thus making the aggregate order flow more informative about $v$ (i.e., $\operatorname{var}\left[P_{P T}^{*} \mid S, S_{p}\right]$ higher).

The dependency of (absolute and relative) price impact and price informativeness on realizations of public news is novel to the literature because, as previously emphasized in section 3.2, it is due to unconventional, Prospect Theory-inspired, state-dependent preferences rather than unconventional representations of agents' beliefs, as in Foster and Viswanathan (1993). This dependency is also important for testing our model's implications, since the econometrician may only observe $S_{p}$ when estimating a traded asset's market liquidity and price efficiency.

\section{Conclusions}

This paper studies the implications of the main features of Tversky and Kahneman's (1979) Prospect Theory — risk seeking over losses, loss aversion, and nonlinear and asymmetric prob-

\footnotetext{
${ }^{17}$ In addition, when neutral public news is released $\left(S_{p}=0\right), E\left[\Delta \lambda_{P T}^{*} \mid S_{p}\right]$ is insensitive to $\mu, \gamma$, and $\sigma_{u}^{2}$ since $S_{p}=E[S]=0$ and $E\left[R\left(\bar{\chi}^{*}, \bar{\chi}\right) \mid S_{p}\right]=1$.
} 
ability weighting (in the spirit of Jullien and Salanié, 2000) — for market liquidity and price informativeness. Our model indicates that risk seeking induces better-informed agents with Prospect theory-inspired preferences to trade more aggressively with their private signals, while loss aversion induces them to use those signals more cautiously. We demonstrate that, because the former effect either prevails on or is reinforced by the latter in equilibrium, the presence of these unconventional speculators in an otherwise standard economy worsens adverse selectionbased market liquidity, makes it countercyclical, and ameliorates price efficiency. We also find that the presence of those speculators affects the extent to which the availability of public news about the traded asset's fundamentals improves market liquidity and price informativeness and makes such improvement procyclical.

These and other predictions of our model are novel and warrant further empirical investigation. Our analysis also raises several unexplored questions. In particular, we note that for simplicity's sake, our model allows for a single round of trading over a single risky asset. Speculators' risk seeking and loss aversion are likely to provide interesting (yet complex) insights for their multiperiod, multiasset trading strategies, especially if combined with that particular form of mental accounting (Thaler, 1980) often displayed by individuals in experimental settings known as narrow framing. ${ }^{18}$ Consistently, the portfolio decisions of such agents and their implications for asset pricing have received increasing attention in the literature (e.g., Levy, De Giorgi, and Hens, 2003; Levy and Levy, 2007; Barberis and Huang, 2008). We look forward to future research that investigates the implications of dynamic portfolio decisions for market liquidity and price efficiency.

\footnotetext{
${ }^{18}$ For instance, this is the circumstance when agents get direct utility from gains and losses of individual assets rather than portfolio fluctuations, as in Barberis and Huang (2001).
} 


\section{Appendix}

Proof of Proposition 1. We prove this statement by using Eq. (3) and properties of conditional normal distributions to solve for $P_{M V}$ from Eq. (4). We begin by observing that the distributional assumptions of Section 2.1 imply that the order flow $\omega=x_{M V}+z$ is normally distributed with mean $E[\omega]=-C_{M V} P$ and variance $\operatorname{var}[\omega]=C_{M V}^{2} \phi^{2} \sigma_{s}^{2}+\sigma_{z}^{2}$, where

$$
C_{M V}=\frac{1}{\alpha \sigma_{v}^{2}(1-\phi)}
$$

Since $\operatorname{cov}[v, \omega]=C_{M V} \phi \sigma_{v}^{2}$, it then follows (e.g., Greene, 1997, p. 90) that:

$$
\begin{aligned}
P & =\frac{\operatorname{cov}[v, \omega]}{\operatorname{var}[\omega]}\{\omega-E[\omega]\} \\
& =\frac{C_{M V} \phi \sigma_{v}^{2}}{C_{M V}^{2} \phi^{2} \sigma_{s}^{2}+\sigma_{z}^{2}-C_{M V}^{2} \phi \sigma_{v}^{2}} \omega .
\end{aligned}
$$

Substituting the expression for $C_{M V}$ (Eq. (A-1)) into Eq. (A-3) and observing that $\phi=\frac{\sigma_{v}^{2}}{\sigma_{v}^{2}+\sigma_{u}^{2}}$ leads to $P_{M V}$ of Eq. (5) with $\lambda_{M V}$ of Eq. (6).

Proof of Corollary 1. The statement of the corollary follows from $\frac{\partial \lambda_{M V}}{\partial \sigma_{v}^{2}}=\frac{1}{\alpha \sigma_{z}^{2} \sigma_{u}^{2}}>0$, $\frac{\partial \lambda_{M V}}{\partial \alpha}=-\frac{\sigma_{v}^{2}}{\alpha^{2} \sigma_{z}^{2} \sigma_{u}^{2}}<0, \frac{\partial \lambda_{M V}}{\partial \sigma_{u}^{2}}=-\frac{\sigma_{v}^{2}}{\alpha \sigma_{z}^{2} \sigma_{u}^{4}}<0$, and $\frac{\partial \lambda_{M V}}{\partial \sigma_{z}^{2}}=-\frac{\sigma_{v}^{2}}{\alpha \sigma_{z}^{4} \sigma_{u}^{2}}<0$.

Proof of Corollary 2. Substitution of Eq. (8) in the expression for $Q_{M V}($ Eq. (7)) implies that

$$
Q_{M V}=\frac{\sigma_{v}^{2}+\sigma_{u}^{2}\left(\alpha^{2} \sigma_{u}^{2} \sigma_{z}^{2}+1\right)}{\sigma_{v}^{2} \sigma_{u}^{2}\left(\alpha^{2} \sigma_{u}^{2} \sigma_{z}^{2}+1\right)}
$$

It then ensues that $\frac{\partial Q_{M V}}{\partial \sigma_{v}^{2}}=-\frac{1}{\sigma_{v}^{4}}<0, \frac{\partial Q_{M V}}{\partial \alpha}=-2 \alpha \frac{\sigma_{z}^{2}}{\left(\alpha^{2} \sigma_{u}^{2} \sigma_{z}^{2}+1\right)^{2}}<0, \frac{\partial Q_{M V}}{\partial \sigma_{u}^{2}}=-\frac{2 \alpha^{2} \sigma_{u}^{2} \sigma_{z}^{2}+1}{\sigma_{u}^{4}\left(\alpha^{2} \sigma_{u}^{2} \sigma_{z}^{2}+1\right)^{2}}<0$, and $\frac{\partial Q_{M V}}{\partial \sigma_{z}^{2}}=-\frac{\alpha^{2}}{\left(\alpha^{2} \sigma_{u}^{2} \sigma_{z}^{2}+1\right)^{2}}<0$.

Proof of Lemma 1. The expression in Eq. (16) is a first-order Taylor series approximation of Eq. (15) where the expansion point is the unconditional mean of $\chi, \bar{\chi}=\frac{P}{\sqrt{\sigma_{v}^{2}(1-\phi)}}$. We begin 
by observing that $x_{M V}$ of Eq. (3) can be expressed as a function of $\chi=\frac{P-\phi S}{\sqrt{\sigma_{v}^{2}(1-\phi)}}$ as

$$
x_{M V}(\chi)=-\frac{\chi}{\alpha \sqrt{\sigma_{v}^{2}(1-\phi)}}
$$

Eq. (A-5) implies that $x_{P T}$ of Eq. (15) can be written as

$$
x_{P T}(\chi)=\frac{1}{\alpha \sqrt{\sigma_{v}^{2}(1-\phi)}}\{-\chi[1+\gamma \Phi(\chi)]-\gamma \psi(\chi)\} .
$$

Since $x_{P T}(\chi)$ is a function of $\chi$ differentiable in a neighborhood of $\bar{\chi}$, it then ensues that

$$
x_{P T}(\chi) \approx x_{P T}(\bar{\chi})+x_{P T}^{\prime}(\bar{\chi})(\chi-\bar{\chi}) .
$$

According to properties of the normal distributions (e.g., Maddala, 1999, p. 367), $\Phi^{\prime}(\chi)=\psi(\chi)$ and $\psi^{\prime}(\chi)=-\chi \psi(\chi)$. The approximation for $x_{M V}$ in Eq. (A-7) then becomes

$$
x_{M V}(\chi) \approx \frac{1}{\alpha \sqrt{\sigma_{v}^{2}(1-\phi)}}\{-\bar{\chi}-\gamma \bar{\chi} \Phi(\bar{\chi})-\gamma \psi(\bar{\chi})-[1+\gamma \Phi(\bar{\chi})](\chi-\bar{\chi})\}
$$

which straightforwardly reduces to Eq. (16).

Proof of Proposition 2. As for Proposition 1, we prove this statement by using Eq. (16) and properties of conditional normal distributions to solve for $P_{P T}$ from Eq. (4). We begin by observing that the definition of $\omega=\mu x_{P T}+(1-\mu) x_{M V}+z$ and Eqs. (3) and (16) imply that

$$
\omega=[1+\mu \gamma \Phi(\bar{\chi})] x_{M V}-\frac{\mu \gamma}{\alpha \sqrt{\sigma_{v}^{2}(1-\phi)}} \psi(\bar{\chi})+z
$$

The distributional assumptions of Section 2.1 imply that $\omega$ is normally distributed with mean $E[\omega]=-C_{P T} P-\frac{\mu \gamma}{\alpha \sqrt{\sigma_{v}^{2}(1-\phi)}} \psi(\bar{\chi})$ and variance $\operatorname{var}[\omega]=C_{P T}^{2} \phi^{2} \sigma_{s}^{2}+\sigma_{z}^{2}$, where

$$
C_{P T}=\frac{1+\mu \gamma \Phi(\bar{\chi})}{\alpha \sigma_{v}^{2}(1-\phi)}
$$

Since $\operatorname{cov}[v, \omega]=C_{P T} \phi \sigma_{v}^{2}$, it then follows from Eq. (A-2) that

$$
P=\frac{C_{P T} \phi \sigma_{v}^{2}}{C_{P T}^{2} \phi^{2} \sigma_{s}^{2}+\sigma_{z}^{2}-C_{P T}^{2} \phi \sigma_{v}^{2}}\left[\omega+C_{P T} P+\frac{\mu \gamma}{\alpha \sqrt{\sigma_{v}^{2}(1-\phi)}} \psi(\bar{\chi})\right] .
$$


Substituting the expression for $C_{P T}$ (Eq. (A-10)) into Eq. (A-11) and observing that $\phi=\frac{\sigma_{v}^{2}}{\sigma_{v}^{2}+\sigma_{u}^{2}}$ leads to the equilibrium $P_{P T}$ as a fixed point of Eq. (17) with $\lambda_{P T}$ of Eq. (18). Such a fixed point exists, since the right side of Eq. (A-11) is a continuous function of $P$. Numerical evaluation shows that, because of properties of $\Phi(\cdot)$ and $\psi(\cdot)$ (in particular, $\Phi^{\prime}(y)=\psi(y)>0$, $\lim _{y \rightarrow+\infty} \Phi(y)=1$, and $\lim _{y \rightarrow-\infty} \Phi(y)=0$, while $\psi^{\prime}(y)=-y \psi(y)$ and $\lim _{y \rightarrow \pm \infty} \psi(y)=0$ and $\psi(0)=\frac{1}{2 \pi}$ ), the right side of Eq. (A-11) is decreasing in $P$ for reasonable parameter values, thus ensuring that the fixed point is unique. Lastly, $\lambda_{P T}>\lambda_{M V}$ since $\Phi(\cdot)$ is bounded between zero and one and both $\gamma$ and $\mu$ are positive.

Proof of Corollary 3. We prove this statement in three steps. First, we observe that given a random variable $y$ with mean $\bar{y}$ and variance $\sigma_{y}^{2}$ and a nonlinear function $g(y)$, firstorder Taylor series expansion around $\bar{y}$ provides a natural approximation of that function's first moment (e.g., Greene, 1997, pp. 66-67), i.e.

$$
E[g(y)] \approx g(\bar{y})
$$

Second, we use the Law of Iterated Expectations (e.g., Greene, 1997, p. 82) and Eq. (4) to show that

$$
E[P]=E_{\omega}[E[v \mid \omega]]=E[v]=0
$$

In addition, it stems from the joint normality of $v$ and $\omega$ that

$$
\operatorname{var}[v \mid \omega]=\sigma_{v}^{2}-\frac{\operatorname{cov}[v, \omega]^{2}}{\operatorname{var}[\omega]}
$$

The Law of Total Variance (e.g., Greene, 1997, p. 83) and Eq. (4) then imply that

$$
\operatorname{var}[P]=\operatorname{var}[E[v \mid \omega]]=\sigma_{v}^{2}-E[\operatorname{var}[v \mid \omega]]=E\left[\frac{\operatorname{cov}[v, \omega]^{2}}{\operatorname{var}[\omega]}\right]
$$


Finally, applying Eq. (A-12) to $\lambda_{P T}$ of Eq. (18) leads to

$$
\begin{aligned}
E\left[\lambda_{P T}\right] & \approx[1+\mu \gamma \Phi(E[\bar{\chi}])] \lambda_{M V} \\
& =\left[1+\mu \gamma \Phi\left(\frac{E[P]}{\sqrt{\sigma_{v}^{2}(1-\phi)}}\right)\right] \lambda_{M V} \\
& =[1+\mu \gamma \Phi(0)] \lambda_{M V},
\end{aligned}
$$

which gives Eq. (19) for $\Phi(0)=\frac{1}{2}$. Since $\operatorname{cov}[v, \omega]=C_{P T} \phi \sigma_{v}^{2}$ and $\operatorname{var}[\omega]=C_{P T}^{2} \phi^{2} \sigma_{s}^{2}+\sigma_{z}^{2}$, where $C_{P T}$ is defined in Eq. (A-10), Eq. (A-15) becomes

$$
\operatorname{var}[P]=E\left[\frac{C_{P T}^{2} \phi^{2} \sigma_{v}^{4}}{C_{P T}^{2} \phi^{2} \sigma_{s}^{2}+\sigma_{z}^{2}}\right] .
$$

Applying Eq. (A-12) to Eq. (A-19) gives

$$
\operatorname{var}[P] \approx \frac{E\left[C_{P T}\right]^{2} \phi^{2} \sigma_{v}^{4}}{E\left[C_{P T}\right]^{2} \phi^{2} \sigma_{s}^{2}+\sigma_{z}^{2}},
$$

where $E\left[C_{P T}\right]=\frac{1+\frac{1}{2} \mu \gamma}{a \sigma_{v}^{2}(1-\phi)}$, i.e., the approximation in Eq. (20).

Proof of Remark 1. Substitution of Eq. (20) in the expression for $Q_{P T}$ (Eq. (21)) implies that

$$
Q_{P T} \approx \frac{\left(1+\frac{1}{2} \mu \gamma\right)^{2}\left(\sigma_{v}^{2}+\sigma_{u}^{2}\right)+\alpha^{2} \sigma_{u}^{4} \sigma_{z}^{2}}{\left(1+\frac{1}{2} \mu \gamma\right)^{2} \sigma_{v}^{2} \sigma_{u}^{2}+\alpha^{2} \sigma_{u}^{4} \sigma_{z}^{2} \sigma_{v}^{2}} .
$$

It then ensues that $\frac{\partial Q_{P T}}{\partial \mu}=\frac{8(2+\mu \gamma) \gamma \sigma_{z}^{2} \alpha^{2}}{\left(4+4 \mu \gamma+\mu^{2} \gamma^{2}+4 \alpha^{2} \sigma_{u}^{2} \sigma_{z}^{2}\right)^{2}}>0, \frac{\partial Q_{P T}}{\partial \gamma}=\frac{8(2+\mu \gamma) \mu \sigma_{z}^{2} \alpha^{2}}{\left(4+4 \mu \gamma+\mu^{2} \gamma^{2}+4 \alpha^{2} \sigma_{u}^{2} \sigma_{z}^{2}\right)^{2}}>0, \frac{\partial Q_{P T}}{\partial \sigma_{v}^{2}}=$ $-\frac{1}{\sigma_{v}^{4}}<0, \frac{\partial Q_{P T}}{\partial \alpha}=-\frac{8 \alpha \sigma_{z}^{2}\left(4+4 \mu \gamma+\mu^{2} \gamma^{2}\right)}{\left(4+4 \mu \gamma+\mu^{2} \gamma^{2}+4 \alpha^{2} \sigma_{u}^{2} \sigma_{z}^{2}\right)^{2}}<0, \frac{\partial Q_{P T}}{\partial \sigma_{u}^{2}}=-\frac{\left(4+4 \mu \gamma+\mu^{2} \gamma^{2}+8 \alpha^{2} \sigma_{u}^{2} \sigma_{z}^{2}\right)\left(4+4 \mu \gamma+\mu^{2} \gamma^{2}\right)}{\sigma_{u}^{4}\left(4+4 \mu \gamma+\mu^{2} \gamma^{2}+4 \alpha^{2} \sigma_{u}^{2} \sigma_{z}^{2}\right)^{2}}<0$, and $\frac{\partial Q_{P T}}{\partial \sigma_{z}^{2}}=-\frac{\alpha^{2}\left(4+4 \mu \gamma+\mu^{2} \gamma^{2}\right)}{\left(\alpha^{2} \sigma_{u}^{2} \sigma_{z}^{2}+1\right)^{2}}<0$.

Proof of Proposition 3. As for Proposition 1, we prove this statement by using Eq. (22) and properties of conditional normal distributions to solve for $P_{M V}^{*}$ from Eq. (23). We begin by observing that the distributional assumptions of Section 4.1 imply that conditional upon observing the public signal $S_{p}$, the order flow $\omega=x_{M V}^{*}+z$ is normally distributed with mean $E\left[\omega \mid S_{p}\right]=-C_{M V}^{*}\left(P-\phi_{p} S_{p}\right)$ and variance $\operatorname{var}\left[\omega \mid S_{p}\right]=\left(C_{M V}^{*} \phi^{*}\right)^{2} \sigma_{s}^{2 *}+\sigma_{z}^{2}$, where

$$
C_{M V}^{*}=\frac{1}{\alpha \sigma_{v}^{2 *}\left(1-\phi^{*}\right)} .
$$


Since $\operatorname{cov}\left[v, \omega \mid S_{p}\right]=C_{M V}^{*} \phi^{*} \sigma_{v}^{2 *}$, it then follows (e.g., Greene, 1997, p. 90) that:

$$
\begin{aligned}
P & =\frac{\operatorname{cov}\left[v, \omega \mid S_{p}\right]}{\operatorname{var}\left[\omega \mid S_{p}\right]}\left\{\omega-E\left[\omega \mid S_{p}\right]\right\} \\
& =\frac{C_{M V}^{*} \phi^{*} \sigma_{v}^{2 *}}{\left(C_{M V}^{*} \phi^{*}\right)^{2} \sigma_{s}^{2 *}+\sigma_{z}^{2}-\left(C_{M V}^{*}\right)^{2} \phi \sigma_{v}^{2 *}} \omega .
\end{aligned}
$$

Substituting the expression for $C_{M V}$ (Eq. (A-22)) into Eq. (A-24) and observing that $\phi^{*}=\frac{\sigma_{v}^{2 *}}{\sigma_{v}^{2 *}+\sigma_{u}^{2}}$ leads to $P_{M V}^{*}$ of Eq. (24) with $\lambda_{M V}^{*}$ of Eq. (25). Lastly, $\lambda_{M V}^{*}<\lambda_{M V}$ since $\phi_{p}>0$.

Proof of Remark 2. It follows from the definition of $\Delta \lambda_{M V}^{*}$ in Eq. (26) and $\phi_{p}=\frac{\sigma_{v}^{2}}{\sigma_{v}^{2}+\sigma_{e}^{2}}$ that $\frac{\partial \Delta \lambda_{M V}^{*}}{\partial \sigma_{v}^{2}}=-\frac{\sigma_{e}^{2}}{\left(\sigma_{v}^{2}+\sigma_{e}^{2}\right)^{2}}<0$ and $\frac{\partial \Delta \lambda_{M V}^{*}}{\partial \sigma_{e}^{2}}=\frac{\sigma_{v}^{2}}{\left(\sigma_{v}^{2}+\sigma_{e}^{2}\right)^{2}}>0$.

Proof of Corollary 4. Substitution of Eq. (27) in the expression for $Q_{M V}^{*}$ in Section 4.1 implies that

$$
Q_{M V}^{*}=\frac{\sigma_{v}^{2 *}+\sigma_{u}^{2}\left(\alpha^{2} \sigma_{u}^{2} \sigma_{z}^{2}+1\right)}{\sigma_{v}^{2 *} \sigma_{u}^{2}\left(\alpha^{2} \sigma_{u}^{2} \sigma_{z}^{2}+1\right)}<Q_{M V}
$$

since $\sigma_{v}^{2 *}=\sigma_{v}^{2} \frac{\sigma_{e}^{2}}{\sigma_{v}^{2}+\sigma_{e}^{2}}<\sigma_{v}^{2}$. It then ensues that $\frac{\partial Q_{M V}^{*}}{\partial \sigma_{e}^{2}}=-\frac{1}{\sigma_{e}^{4}}<0, \frac{\partial Q_{M V}^{*}}{\partial \sigma_{v}^{2}}=-\frac{1}{\sigma_{v}^{4}}<0, \frac{\partial Q_{M V}^{*}}{\partial \alpha}=$ $-\frac{2 \alpha \sigma_{z}^{2}}{\left(\alpha^{2} \sigma_{u}^{2} \sigma_{z}^{2}+1\right)^{2}}<0, \frac{\partial Q_{M V}^{*}}{\partial \sigma_{u}^{2}}=-\frac{2 \alpha^{2} \sigma_{u}^{2} \sigma_{z}^{2}+1}{\sigma_{u}^{4}\left(\alpha^{2} \sigma_{u}^{2} \sigma_{z}^{2}+1\right)^{2}}<0$, and $\frac{\partial Q_{M V}^{*}}{\partial \sigma_{z}^{2}}=-\frac{-4 \alpha^{2}\left(4+4 \mu \gamma+\mu^{2} \gamma^{2}\right)}{\left(4+4 \mu \gamma+\mu^{2} \gamma^{2}+4 \alpha^{2} \sigma_{u}^{2} x\right)^{2}}<0$.

Proof of Proposition 4. As for Proposition 3, we prove this statement by using Eq. (31) and properties of conditional normal distributions to solve for $P_{P T}^{*}$ from Eq. (23). Since $\omega=\mu x_{P T}^{*}+(1-\mu) x_{M V}+z$, Eqs. (22) and (31) imply that

$$
\omega=\left[1+\mu \gamma \Phi\left(\bar{\chi}^{*}\right)\right] x_{M V}^{*}-\frac{\mu \gamma}{\alpha \sqrt{\sigma_{v}^{2 *}\left(1-\phi^{*}\right)}} \psi\left(\bar{\chi}^{*}\right)+z .
$$

The distributional assumptions of Section 4.1 imply that conditional upon observing the public signal $S_{p}, \omega$ is normally distributed with mean $E\left[\omega \mid S_{p}\right]=-C_{P T}^{*}\left(P-\phi_{p} S_{p}\right)-\frac{\mu \gamma}{\alpha \sqrt{\sigma_{v}^{2 *}\left(1-\phi^{*}\right)}} \psi\left(\bar{\chi}^{*}\right)$ and variance $\operatorname{var}\left[\omega \mid S_{p}\right]=\left(C_{P T}^{*} \phi^{*}\right)^{2} \sigma_{s}^{2 *}+\sigma_{z}^{2}$, where

$$
C_{P T}^{*}=\frac{1+\mu \gamma \Phi\left(\bar{\chi}^{*}\right)}{\alpha \sigma_{v}^{2 *}\left(1-\phi^{*}\right)}
$$


Since $\operatorname{cov}\left[v, \omega \mid S_{p}\right]=C_{P T}^{*} \phi^{*} \sigma_{v}^{2 *}$, it then follows from Eq. (A-23) that

$$
\begin{aligned}
P= & \frac{C_{P T}^{*} \phi^{*} \sigma_{v}^{2 *}}{\left(C_{P T}^{*} \phi^{*}\right)^{2} \sigma_{s}^{2 *}+\sigma_{z}^{2}-\left(C_{P T}^{*}\right)^{2} \phi^{*} \sigma_{v}^{2 *}}\left[\omega+C_{P T}^{*}\left(P-\phi_{p} S_{p}\right)\right. \\
& \left.+\frac{\mu \gamma}{\alpha \sqrt{\sigma_{v}^{2 *}\left(1-\phi^{*}\right)}} \psi\left(\bar{\chi}^{*}\right)\right] .
\end{aligned}
$$

Substituting the expression for $C_{P T}$ (Eq. (A-27)) into Eq. (A-28) and observing that $\phi^{*}=\frac{\sigma_{v}^{2 *}}{\sigma_{v}^{2 *}+\sigma_{u}^{2}}$ leads to the equilibrium $P_{P T}^{*}$ as a fixed point of Eq. (32) with $\lambda_{P T}^{*}$ of Eq. (33). Such a fixed point exists, since the right side of Eq. (A-28) is a continuous function of $P$. Numerical evaluation shows that, because of properties of $\Phi(\cdot)$ and $\psi(\cdot)$ (in particular, $\Phi^{\prime}(y)=\psi(y)>0$, $\lim _{y \rightarrow+\infty} \Phi(y)=1$, and $\lim _{y \rightarrow-\infty} \Phi(y)=0$, while $\psi^{\prime}(y)=-y \psi(y)$ and $\lim _{y \rightarrow \pm \infty} \psi(y)=0$ and $\psi(0)=\frac{1}{2 \pi}$ ), the right side of Eq. (A-28) is decreasing in $P$ for reasonable parameter values, thus ensuring that the fixed point is unique. Lastly, numerical evaluation also shows that $\lambda_{P T}^{*}<\lambda_{P T}$ since $\lambda_{M V}^{*}<\lambda_{M V}<\lambda_{P T}$ and $\Phi(\cdot)$ is bounded between zero and one.

Proof of Corollary 5. We prove this statement using arguments similar to those in the proof of Corollary 3. In particular, the Law of Iterated Expectations (e.g., Greene, 1997, p. 82) and Eq. (23) imply that

$$
E\left[P \mid S_{p}\right]=E_{\omega, S_{p}}\left[E\left[v \mid \omega, S_{p}\right]\right]=E\left[v \mid S_{p}\right]=\phi_{p} S_{p}
$$

In addition, it stems from the joint normality of $v$ and $\omega$ conditional upon observing the public signal $S_{p}$ that

$$
\operatorname{var}\left[v \mid \omega, S_{p}\right]=\sigma_{v}^{2 *}-\frac{\operatorname{cov}\left[v, \omega \mid S_{p}\right]^{2}}{\operatorname{var}\left[\omega \mid S_{p}\right]}
$$

The Law of Total Variance (e.g., Greene, 1997, p. 83) and Eq. (23) then imply that

$$
\operatorname{var}\left[P \mid S_{p}\right]=\operatorname{var}\left[E\left[v \mid \omega, S_{p}\right]\right]=\sigma_{v}^{2 *}-E_{S_{p}}\left[\operatorname{var}\left[v \mid \omega, S_{p}\right]\right]=E_{S_{p}}\left[\frac{\operatorname{cov}\left[v, \omega \mid S_{p}\right]^{2}}{\operatorname{var}\left[\omega \mid S_{p}\right]}\right] .
$$


Applying Eq. (A-29) to $\lambda_{P T}^{*}$ of Eq. (33) then leads to

$$
\begin{aligned}
E\left[\lambda_{P T}^{*} \mid S_{p}\right] & \approx\left[1+\mu \gamma \Phi\left(E\left[\bar{\chi}^{*}\right]\right)\right] \lambda_{M V}^{*} \\
& =\left[1+\mu \gamma \Phi\left(\frac{E\left[P \mid S_{p}\right]-\phi_{p} S_{p}}{\sqrt{\sigma_{v}^{2 *}\left(1-\phi^{*}\right)}}\right)\right] \lambda_{M V}^{*} \\
& =[1+\mu \gamma \Phi(0)] \lambda_{M V}^{*},
\end{aligned}
$$

which gives Eq. (35) for $\Phi(0)=\frac{1}{2}$. Since $\operatorname{cov}\left[v, \omega \mid S_{p}\right]=C_{P T}^{*} \phi^{*} \sigma_{v}^{2 *}$ and $\operatorname{var}\left[\omega \mid S_{p}\right]=\left(C_{P T}^{*} \phi^{*}\right)^{2} \sigma_{s}^{2 *}+$ $\sigma_{z}^{2}$, where $C_{P T}^{*}$ is defined in Eq. (A-27), Eq. (A-31) becomes

$$
\operatorname{var}\left[P \mid S_{p}\right]=E_{S_{p}}\left[\frac{\left(C_{P T}^{*} \phi^{*}\right)^{2}\left(\sigma_{v}^{2 *}\right)^{2}}{\left(C_{P T}^{*} \phi^{*}\right)^{2} \sigma_{s}^{2 *}+\sigma_{z}^{2}}\right] .
$$

Applying Eq. (A-12) to Eq. (A-35) gives

$$
\operatorname{var}\left[P \mid S_{p}\right] \approx \frac{E\left[C_{P T}^{*} \mid S_{p}\right]^{2}\left(\phi^{*} \sigma_{v}^{2 *}\right)^{2}}{E\left[C_{P T}^{*} \mid S_{p}\right]^{2}\left(\phi^{*}\right)^{2} \sigma_{s}^{2 *}+\sigma_{z}^{2}},
$$

where $E\left[C_{P T}^{*} \mid S_{p}\right]=\frac{1+\frac{1}{2} \mu \gamma}{a \sigma_{v}^{*}\left(1-\phi^{*}\right)}$, i.e., the approximation in Eq. (36). It then ensues straightforwardly from Eqs. (27), (A-20), and (A-36), and $\sigma_{v}^{2 *}<\sigma_{v}^{2}$ that $\operatorname{var}\left[P_{P T}^{*} \mid S_{p}\right]>\operatorname{var}\left[P_{M V}^{*} \mid S_{p}\right]<$ and $\operatorname{var}\left[P_{M V}^{*} \mid S_{p}\right]<\operatorname{var}\left[P_{P T}\right]$. Finally, Eqs. (34), (A-12). and (A-29) imply that

$$
\begin{aligned}
E\left[\Delta \lambda_{P T}^{*} \mid S_{p}\right] & =E_{S_{p}}\left[\frac{1+\mu \gamma \Phi\left(\bar{\chi}^{*}\right)}{1+\mu \gamma \Phi(\bar{\chi})}\right]\left(1-\phi_{p}\right)-1 \\
& =E_{S_{p}}\left[\frac{1+\mu \gamma \Phi\left(\frac{P-\phi_{p} S_{p}}{\sqrt{\sigma_{v}^{*}\left(1-\phi^{*}\right)}}\right)}{1+\mu \gamma \Phi\left(\frac{P}{\sqrt{\sigma_{v}^{2}(1-\phi)}}\right)}\right]\left(1-\phi_{p}\right)-1 \\
& \approx\left[\frac{1+\mu \gamma \Phi\left(\frac{E\left[P \mid S_{p}\right]-\phi_{p} S_{p}}{\sqrt{\sigma_{v}^{2 *}\left(1-\phi^{*}\right)}}\right)}{1+\mu \gamma \Phi\left(\frac{E\left[P \mid S_{p}\right]}{\sqrt{\sigma_{v}^{2}(1-\phi)}}\right)}\right]\left(1-\phi_{p}\right)-1,
\end{aligned}
$$

i.e., the expression in Eq. (37).

Proof of Corollary 6. Substitution of Eq. (36) in the expression for $Q_{P T}^{*}$ in Section 4.2 implies that

$$
Q_{P T}^{*} \approx \frac{\left(1+\frac{1}{2} \mu \gamma\right)^{2}\left(\sigma_{v}^{2 *}+\sigma_{u}^{2}\right)+\alpha^{2} \sigma_{u}^{4} \sigma_{z}^{2}}{\left(1+\frac{1}{2} \mu \gamma\right)^{2} \sigma_{v}^{2 *} \sigma_{u}^{2}+\alpha^{2} \sigma_{u}^{4} \sigma_{z}^{2} \sigma_{v}^{2 *}}
$$


It then ensues that $\frac{\partial Q_{P T}^{*}}{\partial \mu}=\frac{8(2+\mu \gamma) \gamma \sigma_{z}^{2} \alpha^{2}}{\left(4+4 \mu \gamma+\mu^{2} \gamma^{2}+4 \alpha^{2} \sigma_{u}^{2} \sigma_{z}^{2}\right)^{2}}>0, \frac{\partial Q_{P T}^{*}}{\partial \gamma}=\frac{8(2+\mu \gamma) \mu \sigma_{z}^{2} \alpha^{2}}{\left(4+4 \mu \gamma+\mu^{2} \gamma^{2}+4 \alpha^{2} \sigma_{u}^{2} \sigma_{z}^{2}\right)^{2}}>0, \frac{\partial Q_{P T}^{*}}{\partial \sigma_{v}^{2}}=$ $-\frac{\sigma_{v}^{2}+\sigma_{e}^{2}}{\sigma_{e}^{2} \sigma_{v}^{4}}<0, \frac{\partial Q_{P T}^{*}}{\partial \alpha}=-\frac{8 \alpha \sigma_{z}^{2}\left(4+4 \mu \gamma+\mu^{2} \gamma^{2}\right)}{\left(4+4 \mu \gamma+\mu^{2} \gamma^{2}+4 \alpha^{2} \sigma_{u}^{2} \sigma_{z}^{2}\right)^{2}}<0, \frac{\partial Q_{P T}^{*}}{\partial \sigma_{u}^{2}}=-\frac{\left(4+4 \mu \gamma+\mu^{2} \gamma^{2}+8 \alpha^{2} \sigma_{u}^{2} \sigma_{z}^{2}\right)\left(4+4 \mu \gamma+\mu^{2} \gamma^{2}\right)}{\sigma_{u}^{4}\left(4+4 \mu \gamma+\mu^{2} \gamma^{2}+4 \alpha^{2} \sigma_{u}^{2} \sigma_{z}^{2}\right)^{2}}<0$, and $\frac{\partial Q_{P T}^{*}}{\partial \sigma_{z}^{2}}=-\frac{\alpha^{2}\left(4+4 \mu \gamma+\mu^{2} \gamma^{2}\right)}{\left(\alpha^{2} \sigma_{u}^{2} \sigma_{z}^{2}+1\right)^{2}}<0$.

Proof of Remark 3. Eq. (37) and the observation that $\phi=\frac{\sigma_{v}^{2}}{\sigma_{v}^{2}+\sigma_{u}^{2}}$ and $\phi_{p}=\frac{\sigma_{v}^{2}}{\sigma_{v}^{2}+\sigma_{e}^{2}}$ lead to

$$
E\left[\Delta \lambda_{P T}^{*} \mid S_{p}\right] \approx \frac{\sigma_{e}^{2}\left(1+\frac{1}{2} \mu \gamma\right)}{\left(\sigma_{v}^{2}+\sigma_{e}^{2}\right)\left[1+\mu \gamma \Phi\left(\frac{\sigma_{v}^{2}}{\sigma_{v}^{2}+\sigma_{e}^{2}} \sqrt{\frac{\sigma_{v}^{2}+\sigma_{u}^{2}}{\sigma_{v}^{2} \sigma_{u}^{u}}} S_{p}\right)\right]}-1
$$

Eq. (A-41) and properties of $\Phi(\cdot)$ then imply that

$$
\begin{aligned}
& \frac{\partial E\left[\Delta \lambda_{P T}^{*} \mid S_{p}\right]}{\partial \mu}=\frac{\gamma \sigma_{e}^{2}\left[\frac{1}{2}-\Phi\left(\frac{\sigma_{v}^{2}}{\sigma_{v}^{2}+\sigma_{e}^{2}} \sqrt{\frac{\sigma_{v}^{2}+\sigma_{u}^{2}}{\sigma_{v}^{2} \sigma_{u}^{2}}} S_{p}\right)\right]}{\left(\sigma_{v}^{2}+\sigma_{e}^{2}\right)\left[1+\mu \gamma \Phi\left(\frac{\sigma_{v}^{2}}{\sigma_{v}^{2}+\sigma_{e}^{2}} \sqrt{\frac{\sigma_{v}^{2}+\sigma_{u}^{2}}{\sigma_{v}^{2} \sigma_{u}^{2}}} S_{p}\right)\right]^{2}}\left\{\begin{array}{ll}
>0 & \text { if } S_{p}<0 \\
<0 & \text { if } S_{p}>0
\end{array},\right. \\
& \frac{\partial E\left[\Delta \lambda_{P T}^{*} \mid S_{p}\right]}{\partial \gamma}=\frac{\mu \sigma_{e}^{2}\left[\frac{1}{2}-\Phi\left(\frac{\sigma_{v}^{2}}{\sigma_{v}^{2}+\sigma_{e}^{2}} \sqrt{\frac{\sigma_{v}^{2}+\sigma_{u}^{2}}{\sigma_{v}^{2} \sigma_{u}^{2}}} S_{p}\right)\right]}{\left(\sigma_{v}^{2}+\sigma_{e}^{2}\right)\left[1+\mu \gamma \Phi\left(\frac{\sigma_{v}^{2}}{\sigma_{v}^{2}+\sigma_{e}^{2}} \sqrt{\frac{\sigma_{v}^{2}+\sigma_{u}^{2}}{\sigma_{v}^{2} \sigma_{u}^{2}}} S_{p}\right)\right]^{2}}\left\{\begin{array}{ll}
>0 & \text { if } S_{p}<0 \\
<0 & \text { if } S_{p}<0
\end{array},\right. \\
& \frac{\partial E\left[\Delta \lambda_{P T}^{*} \mid S_{p}\right]}{\partial \sigma_{u}^{2}}=\frac{\sigma_{e}^{2} \sigma_{v}^{4}\left(1+\frac{1}{2} \mu \gamma\right) \mu \gamma \psi\left(\frac{\sigma_{v}^{2}}{\sigma_{v}^{2}+\sigma_{e}^{2}} \sqrt{\frac{\sigma_{v}^{2}+\sigma_{u}^{2}}{\sigma_{v}^{2} \sigma_{u}^{2}}} S_{p}\right) S_{p}}{2 \sigma_{v}^{2}\left(\sigma_{v}^{2}+\sigma_{e}^{2}\right)^{2}\left(\sigma_{v}^{2}+\sigma_{u}^{2}\right) \sqrt{\frac{\sigma_{v}^{2} \sigma_{u}^{2}}{\sigma_{v}^{2}+\sigma_{u}^{u}}}\left[1+\mu \gamma \Phi\left(\frac{\sigma_{v}^{2}}{\sigma_{v}^{2}+\sigma_{e}^{2}} \sqrt{\frac{\sigma_{v}^{2}+\sigma_{u}^{2}}{\sigma_{v}^{2} \sigma_{u}^{u}}} S_{p}\right)\right]^{2}} \\
& =\left\{\begin{array}{cc}
<0 & \text { if } S_{p}<0 \\
>0 & \text { if } S_{p}>0
\end{array},\right.
\end{aligned}
$$

while numerical evaluation of their more involved expressions shows that $\frac{\partial E\left[\Delta \lambda_{P T}^{*} \mid S_{p}\right]}{\partial \sigma_{v}^{2}}<0$ and $\frac{\partial E\left[\Delta \lambda_{P T}^{*} \mid S_{p}\right]}{\partial \sigma_{e}^{2}}>0$, where

$$
\begin{aligned}
\frac{\partial E\left[\Delta \lambda_{P T}^{*} \mid S_{p}\right]}{\partial \sigma_{v}^{2}}= & -\frac{\sigma_{e}^{2}}{\sigma_{v}^{2}+\sigma_{e}^{2}} \frac{1+\frac{1}{2} \mu \gamma}{1+\mu \gamma \Phi\left(\frac{\sigma_{v}^{2}}{\sigma_{v}^{2}+\sigma_{e}^{2}} \sqrt{\frac{\sigma_{v}^{2}+\sigma_{u}^{2}}{\sigma_{v}^{2} \sigma_{u}^{2}}} S_{p}\right)}\left\{\frac{\frac{1}{2} \mu \gamma \psi\left(\frac{\sigma_{v}^{2}}{\sigma_{v}^{2}+\sigma_{e}^{2}} \sqrt{\frac{\sigma_{v}^{2}+\sigma_{u}^{2}}{\sigma_{v}^{2} \sigma_{u}^{2}}} S_{p}\right)}{1+\mu \gamma \Phi\left(\frac{\sigma_{v}^{2}}{\sigma_{v}^{2}+\sigma_{e}^{2}} \sqrt{\frac{\sigma_{v}^{2}+\sigma_{2}^{2}}{\sigma_{v}^{2} \sigma_{u}}} S_{p}\right)}\right. \\
& \left.\frac{2 \sigma_{e}^{2} \sigma_{v}^{2}+\sigma_{e}^{2} \sigma_{u}^{2}-\sigma_{v}^{2} \sigma_{u}^{2}}{\left(\sigma_{v}^{2}+\sigma_{e}^{2}\right)^{2} \sigma_{v} \sigma_{u} \sqrt{\sigma_{v}^{2}+\sigma_{u}^{2}}} S_{p}+\frac{1}{\sigma_{v}^{2}+\sigma_{e}^{2}}\right\} \\
\frac{\partial E\left[\Delta \lambda_{P T}^{*} \mid S_{p}\right]}{\partial \sigma_{e}^{2}}= & \frac{\sigma_{v}^{2}}{\sigma_{v}^{2}+\sigma_{e}^{2}} \frac{1+\frac{1}{2} \mu \gamma}{1+\mu \gamma \Phi\left(\frac{\sigma_{v}^{2}}{\sigma_{v}^{2}+\sigma_{e}^{2}} \sqrt{\frac{\sigma_{v}^{2}+\sigma_{u}^{2}}{\sigma_{v}^{2} \sigma_{u}^{2}}} S_{p}\right)}\left\{\frac{\sigma_{e}^{2} \mu \gamma \psi\left(\frac{\sigma_{v}^{2}}{\sigma_{v}^{2}+\sigma_{e}^{2}} \sqrt{\frac{\sigma_{v}^{2}+\sigma_{u}^{2}}{\sigma_{v}^{2} \sigma_{u}^{2}}} S_{p}\right)}{1+\mu \gamma \Phi\left(\frac{\sigma_{v}^{2}}{\sigma_{v}^{2}+\sigma_{e}^{2}} \sqrt{\frac{\sigma_{v}^{2}+\sigma_{u}^{2}}{\sigma_{v}^{2} \sigma_{u}^{2}}} S_{p}\right)}\right. \\
& \left.\frac{\sqrt{\sigma_{v}^{2}+\sigma_{u}^{2}}}{\left(\sigma_{v}^{2}+\sigma_{e}^{2}\right)^{2} \sigma_{v} \sigma_{u}} S_{p}+\frac{1}{\sigma_{v}^{2}+\sigma_{e}^{2}}\right\},
\end{aligned}
$$


since $\Phi(y)$ is increasing in $y$ and bounded between zero and one, while $\lim _{y \rightarrow \pm \infty} \psi(y)=0$ and $\psi(0)=\frac{1}{2 \pi}$.

\section{References}

Admati, A., and Pfleiderer, P., 1988, A Theory of Intraday Trading Patterns: Volume and Price Variability, Review of Financial Studies, 1, 3-40.

Aït-Sahalia, Y., and Brandt, M., 2001, Variable Selection for Portfolio Choice, Journal of Finance, 56, 1297-1351.

Andersen, T., and Bollerslev, T., 1998, Deutsche Mark-Dollar Volatility: Intraday Activity Patterns, Macroeconomic Announcements, and Longer Run Dependencies, Journal of Finance, 53, 219-265.

Barberis, N., and Huang, M., 2001, Mental Accounting, Loss Aversion, and Individual Stock Returns, Journal of Finance, 56, 1247-1292.

Barberis, N., and Huang, M., 2008, Stocks as Lotteries: The Implications of Probability Weighting for Security Prices, American Economic Review, forthcoming.

Barberis, N., Huang, M., and Santos, T., 2001, Prospect Theory and Asset Prices, Quarterly Journal of Economics, 116, 1-53.

Barberis, N., Huang, M., and Thaler, R., 2006, Individual Preferences, Monetary Gambles, and Stock Market Participation: A Case for Narrow Framing, American Economic Review, 96, 10691090.

Barberis, N., Schleifer, A., and Vishny, R., 1998, A Model of Investor Sentiment, Journal of Financial Economics, 49, 307-343. 
Barberis, N., and Xiong, W., 2006, What Drives the Disposition Effect? An Analysis of a Long-standing Preference-based Explanation, Journal of Finance, forthcoming.

Berkelaar, A., Kouwenberg, R., and Post, T., 2004, Optimal Portfolio Choice under Loss Aversion, Review of Economics and Statistics, 86, 973-987.

Benartzi, S., and Thaler, R., 1995, Myopic Loss Aversion and the Equity Premium Puzzle, Quarterly Journal of Economics, 110, 73-92.

Berry, T., and Howe, K., 1994, Public Information Arrival, Journal of Finance, 49, 1331-1346.

Blanchard, O., and Kahn, C., 1980, The Solution to Linear Difference Models under Rational Expectations, Econometrica, 48, 1305-1311.

Boyd, J., Hu, J., and Jagannathan, R., 2005, The Stock Market's Reaction to Unemployment News: Why Bad News Is Usually Good For Stocks, Journal of Finance, 60, 649-672.

Brandt, M., and Kavajecz, K., 2004, Price Discovery in the U.S. Treasury Market: The Impact of Order Flow and Liquidity on the Yield Curve, Journal of Finance, 59, 2623-2654.

Camerer, C., 2000, Prospect Theory in the Wild: Evidence from the Field, in Kahneman, D., and Tversky, A. (Eds.), Choices, Values, and Frames, Cambridge University Press, pp. 288-300.

Chordia, T., Roll, R., and Subrahmanyam, A., 2001, Market Liquidity and Trading Activity, Journal of Finance, 56, 501-530.

Chordia, T., Shivakumar, L., and Subrahmanyam, A., 2004, Liquidity Dynamics Across Small and Large Firms, Economic Notes, 33, 111-143.

Daniel, K., Hirshleifer, D., and Subrahmanyam, A., 2001, Covariance Risk, Mispricing, and the Cross Section of Security Returns, Journal of Finance, 56, 921-965. 
Deuskar, P., 2008, Market Structure and the Effect of Sentiment on Liquidity, Working Paper, University of Illinois.

Diamond, D., and Verrecchia, R., 1981, Information Aggregation in a Noisy Rational Expectations Economy, Journal of Financial Economics, 9, 221-235.

Diamond, D., and Verrecchia, R., 1991, Disclosure, Liquidity, and the Cost of Capital, Journal of Finance, 46, 1325-1359.

Evans, M., and Lyons, R., 2003, How is Macro News Transmitted to Exchange Rates, Journal of Financial Economics, forthcoming.

Fleming, M., and Remolona, E., 1999, Price Formation and Liquidity in the U.S. Treasury Market: The Response to Public Information, Journal of Finance, 54, 1901-1915.

Foster, F., and Viswanathan, S., 1990, A Theory of the Interday Variations in Volume, Variance, and Trading Costs in Securities Markets, Review of Financial Studies, 3, 593-624.

Foster, F., and Viswanathan, S., 1993, The Effect of Public Information and Competition on Trading Volume and Price Volatility, Review of Financial Studies, 6, 23-56.

Gomes, F., 2005, Portfolio Choice and Trading Volume with Loss-Averse Investors, Journal of Business, 78, 675-706.

Green, C., 2004, Economic News and the Impact of Trading on Bond Prices, Journal of Finance, 59, 1201-1233.

Greene, W., 1997, Econometric Analysis, Prentice Hall.

Griffin, J., Nardari, F, and Stulz, R., 2007, Di Investors Trade More When Stocks Have Performed Well? Evidence from 46 Countries, Review of Financial Studies, 20, 905-951. 
Grinblatt, M., and Han, B., 2005, Prospect Theory, Mental Accounting, and Momentum, Journal of Financial Economics, 78, 311-339.

Grossman, S., and Stiglitz, J., 1980, On the Impossibility of Informationally Efficient Markets, American Economic Review, 70, 393-408.

Hasbrouck, J., 2007, Empirical Market Microstructure, Oxford University Press.

Hong, H., and Stein, J., 1999, A Unified Theory of Underreaction, Momentum Trading, and Overreaction in Asset Markets, Journal of Finance, 54, 2143-2184.

Huang, C., and Litzenberger, R., 1988, Foundations for Financial Economics, Prentice Hall.

Jullien, B., and Salanié, B., 2000, Estimating Preferences under Risk: The Case of Racetrack Bettors, Journal of Political Economy, 108, 503-530.

Kahneman, D., and Tversky, A., 1979, Prospect Theory: An Analysis of Decision under Risk, Econometrica, 47, 263-291.

Kamara, A., Lou, X., and Sadka, R., 2007, The Divergence of Liquidity Commonality in the Cross-Section of Stocks, Journal of Financial Economics, forthcoming.

Kim, O., and Verrecchia, R., 1994, Market Liquidity and Volume around Earnings Announcements, Journal of Accounting and Economics, 17, 41-67.

Kim, O., and Verrecchia, R., 1997, Pre-Announcement and Event-Period Private Information, Journal of Accounting and Economics, 24, 395-419.

Klein, P., 2000, Using the Generalized Schur Form to Solve a Multivariate Linear Rational Expectations Model, Journal of Economic Dynamics and Control, 24, 1405-1423.

Kogan, L., Ross, S., Wang, J., and Westerfield, M., 2006, The Price Impact and Survival of Irrational Traders, Journal of Finance, 61, 195-229. 
Kovalenkov, A., and Vives, X., 2007, Competitive Rational Expectations Equilibria without Apology, Working Paper, IESE Business School.

Kyle, A., 1985, Continuous Auctions and Insider Trading, Econometrica, 53, 1315-1335.

Kyle, A., Ou-Yang, H., and Xiong, W., 2006, Prospect Theory and Liquidation Decisions, Journal of Economic Theory, 129, 273-288.

Levy, H., De Giorgi, E., and Hens, T., 2003, Two Paradigms and Two Nobel Prizes in Economics: A Contradiction or Coexistence?, Working Paper, Hebrew University.

Levy, H., and Levy, M., 2004, Prospect Theory and Mean-Variance Analysis, Review of Financial Studies, 17, 1015-1041.

Lombardo, G., and Sutherland, A., 2007, Computing Second-Order Accurate Solutions for Rational Expectations Models Using Linear Solution Methods, Journal of Economic Dynamics and Control, 31, 515-530.

Maddala, G., 1999, Limited-Dependent and Qualitative Variables in Econometrics, Cambridge University Press.

McKelvey, R., and Page, T., 1990, Public and Private Information: An Experimental Study of Information Pooling, Econometrica, 58, 1321-1339.

Nofsinger, J., 2005, The Psychology of Investing, Pearson Prentice Hall.

O'Hara, M., 1995, Market Microstructure Theory, Blackwell Publishers.

Pasquariello, P., and Vega, C., 2007, Informed and Strategic Order Flow in the Bond Markets, Review of Financial Studies, 20, 1975-2019.

Sims, C., 2000, Solving Linear Rational Expectations Models, Working Paper, Princeton University. 
Subrahmanyam, A., 1991, Risk Aversion, Market Liquidity, and Price Efficiency, Review of Financial Studies, 4, 417-441.

Thaler, R., 1980, Toward a Positive Theory of Consumer Choice, Journal of Economic Behavior and Organization, 1, 39-60.

Tversky, A., and Kahneman, D., 1992, Advances in Prospect Theory: Cumulative Representation of Uncertainty, Journal of Risk and Uncertainty, 5, 297-323.

Verrecchia, R., 1982, Information Acquisition in a Noisy Rational Expectations Economy, Econometrica, 50, 1415-1430.

Vives, X., 2008, Information and Learning in Markets, Princeton University Press. 


\section{Figure 1. Prospect Theory-Inspired Preferences}

In this figure we plot, under the assumption that $P=0$, realizations of the Prospect Theory-inspired utility function for PT speculators of Section 3.1 (i.e., $U_{M V+P T}=\pi-\frac{1}{2} \alpha \pi^{2}+V(\pi)$ from Eq. (10), solid line) for $\alpha=1$ and $\gamma=3$, of its risk-neutral addendum in losses (i.e., $V(\pi)$ of Eq. (11), dashed line), of the piecewise power utility function proposed by Tversky and Kahneman (1992) ( $U_{P T}$ of Eq. (9), dotted line), and of the value function of MV speculators (i.e., $U_{M V}=\pi-\frac{1}{2} \alpha \pi^{2}$ from Eq. (2), thin line) over the domain of trading profits $\pi$.

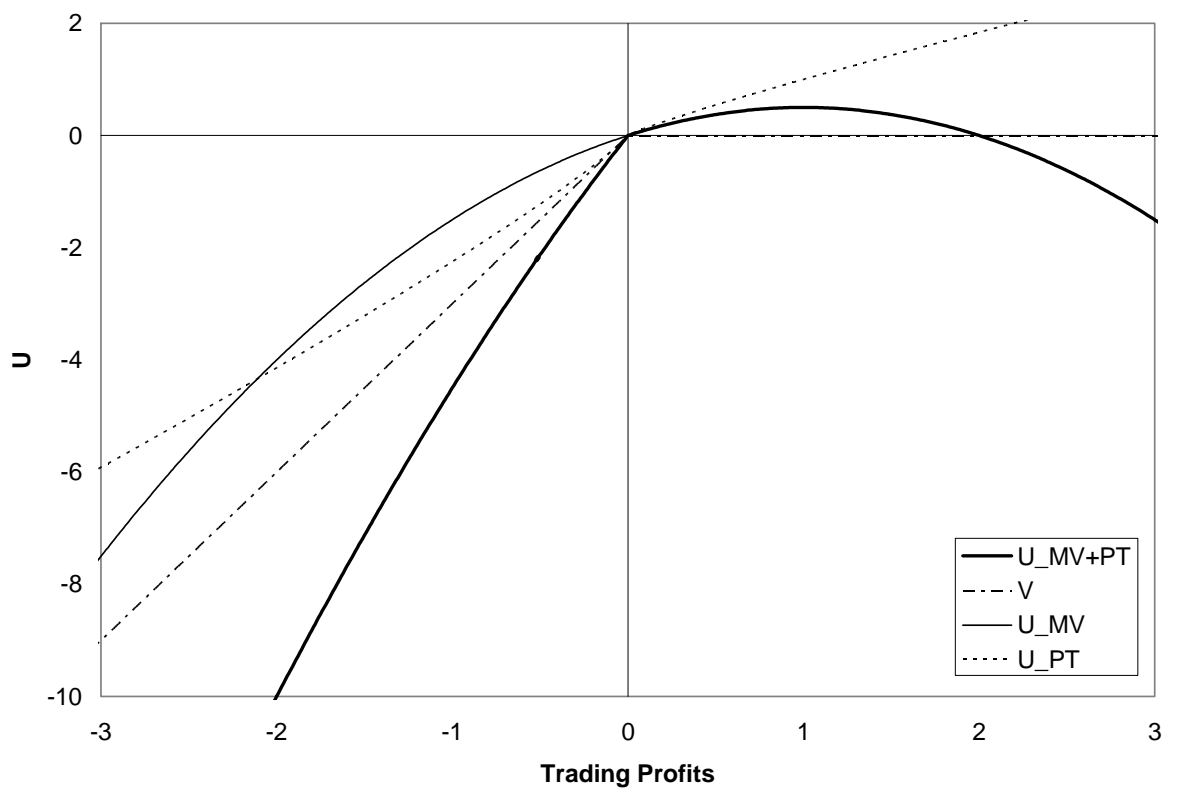




\section{Figure 2. Prospect Theory-Inspired Demand Function}

In this figure we plot PT and MV speculators' optimal demand schedules $x_{P T}$ (Eq. (15), solid line) and $x_{M V}$ (Eq. (3), thin line), respectively, as well as $(1+\gamma) x_{M V}$ (dashed line) and the first-order representation of PT speculators' optimal trading strategy in Lemma 1 (Eq. (16), thin gray line) over the domain of $P$ for a private signal $S=0$ when $\sigma_{v}^{2}=\sigma_{u}^{2}=\sigma_{z}^{2}=1, \alpha=1$, and $\gamma=3$.

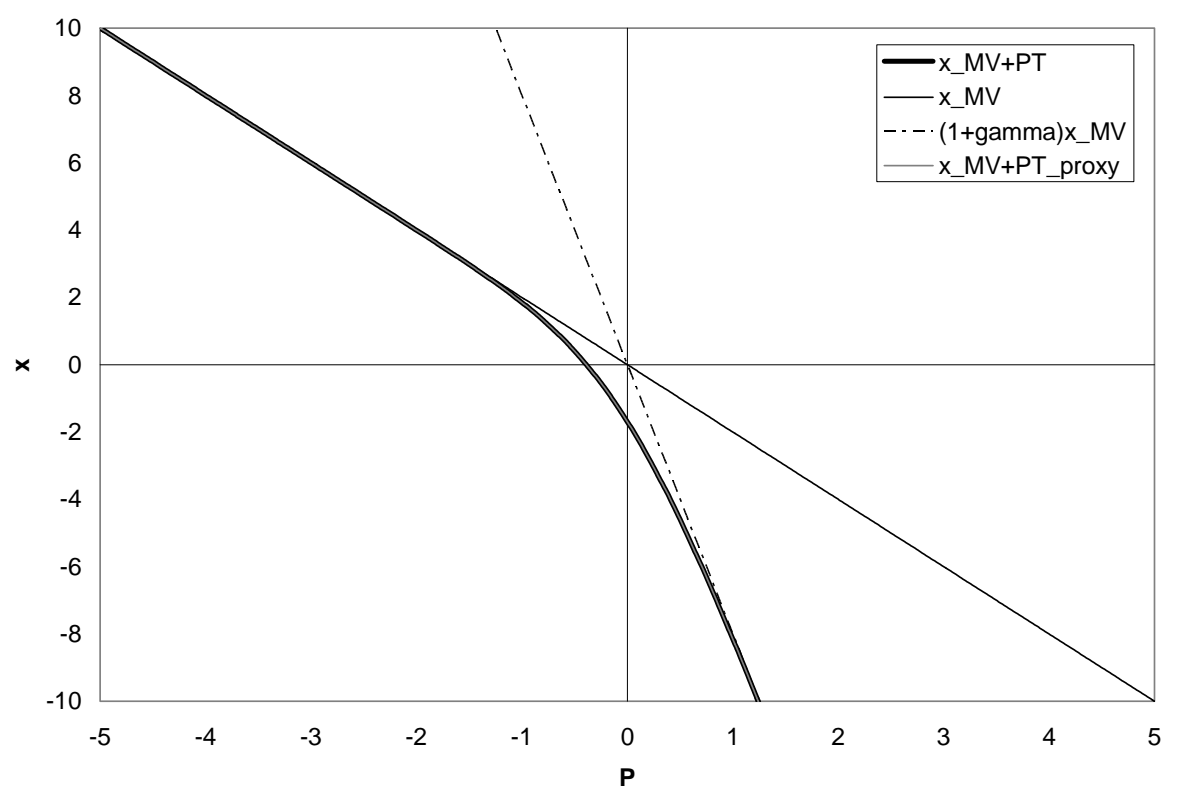




\section{Figure 3. Prospect Theory and Market Liquidity}

In this figure we plot, by virtue of numerical integration, the average equilibrium price impact $\lambda_{P T}$ of Proposition $2\left(E\left[\lambda_{P T} \mid S\right]\right.$, solid line, right axis, upper panel), PT speculators' optimal trading activity $\left(E\left[x_{P T} \mid S\right]\right.$, thin line, left axis, upper panel), subjective cumulative trading loss probability $(E[\Phi(\bar{\chi}) \mid S]$, solid gray line, lower panel) and marginal trading loss probability $(E[\psi(\bar{\chi}) \mid S]$, thin gray line, lower panel) with respect to all possible noise trading shocks $z$ over the domain of $S$ when $\sigma_{v}^{2}=\sigma_{u}^{2}=\sigma_{z}^{2}=1, \alpha=1, \mu=0.1$, and $\gamma=3$, as well as the price impact of Proposition 1 (i.e., $\lambda_{M V}$, dotted line, right axis) and MV speculators' average optimal trading activity $\left(E\left[x_{M V} \mid S\right]\right.$, thin line, left axis) when $\mu=0$.
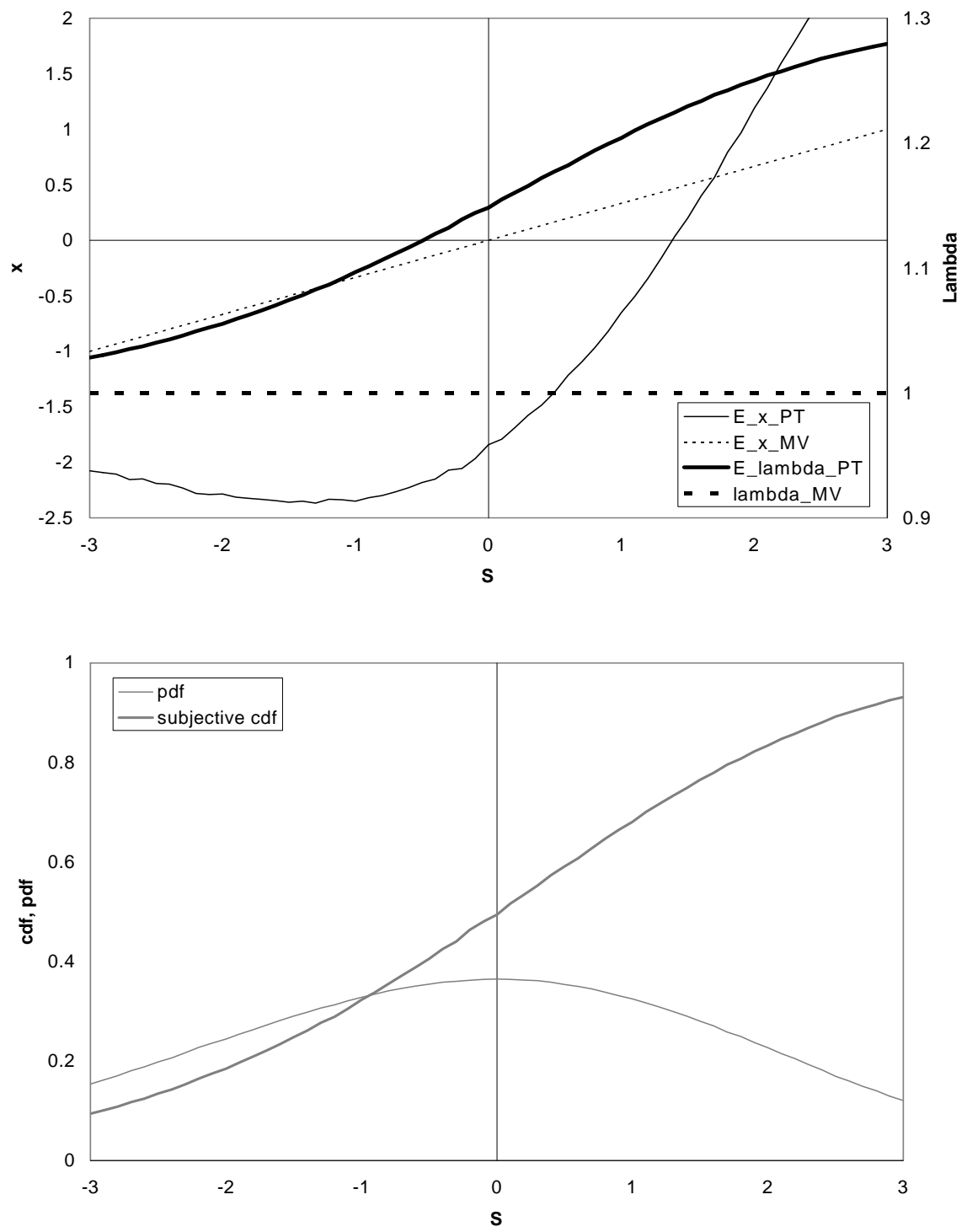


\section{Figure 4. Optimal Trading and a Public Signal}

In this figure we plot PT and MV speculators' optimal demand schedules $x_{P T}^{*}$ (Eq. (30), solid line) and $x_{M V}^{*}$ (Eq. (22), dotted line), respectively, and the first-order representation of PT speculators' optimal trading strategy (Eq. (31), thin gray line) when a public signal $S_{p}=0$ is available, as well as $x_{M V}$ (Eq. (3), thin line) and $x_{P T}$ (Eq. (15), dashed gray line) when $S_{p}$ is unavailable, over the domain of $P$ for a private signal $S=0$ when $\sigma_{v}^{2}=\sigma_{u}^{2}=\sigma_{z}^{2}=1, \alpha=1$, and $\gamma=3$.

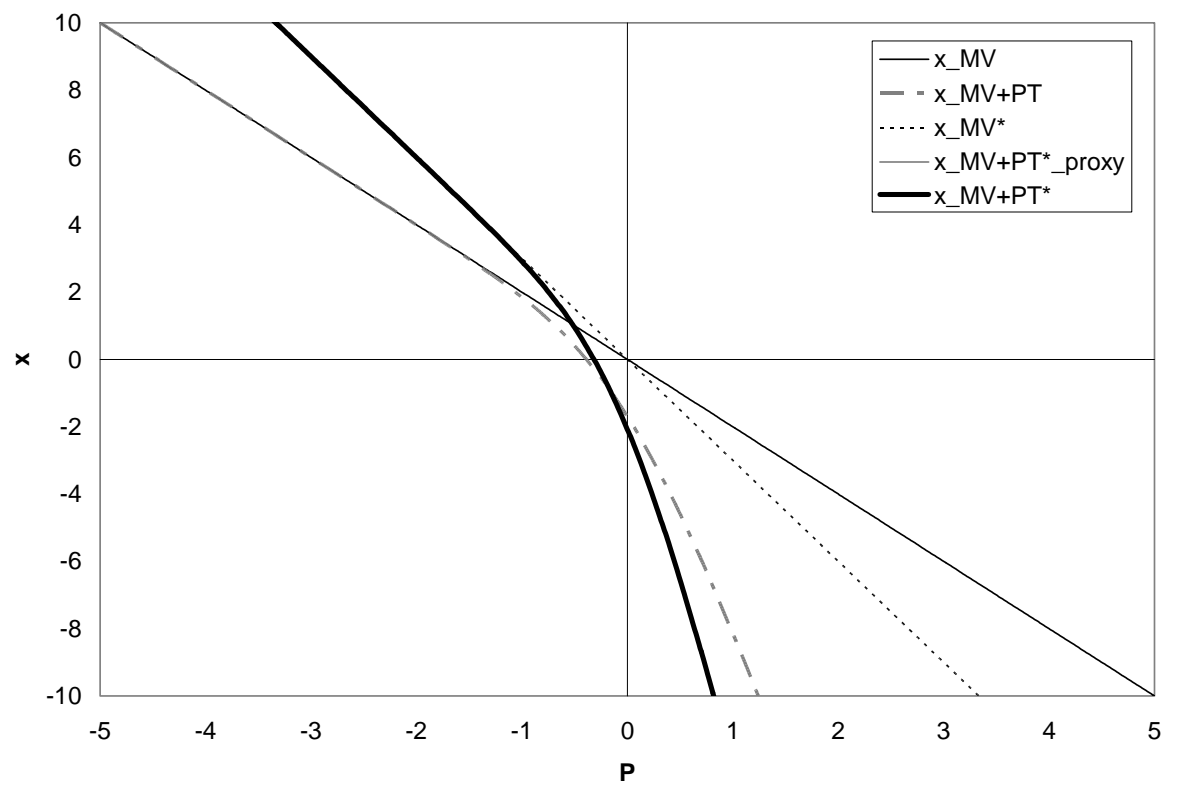




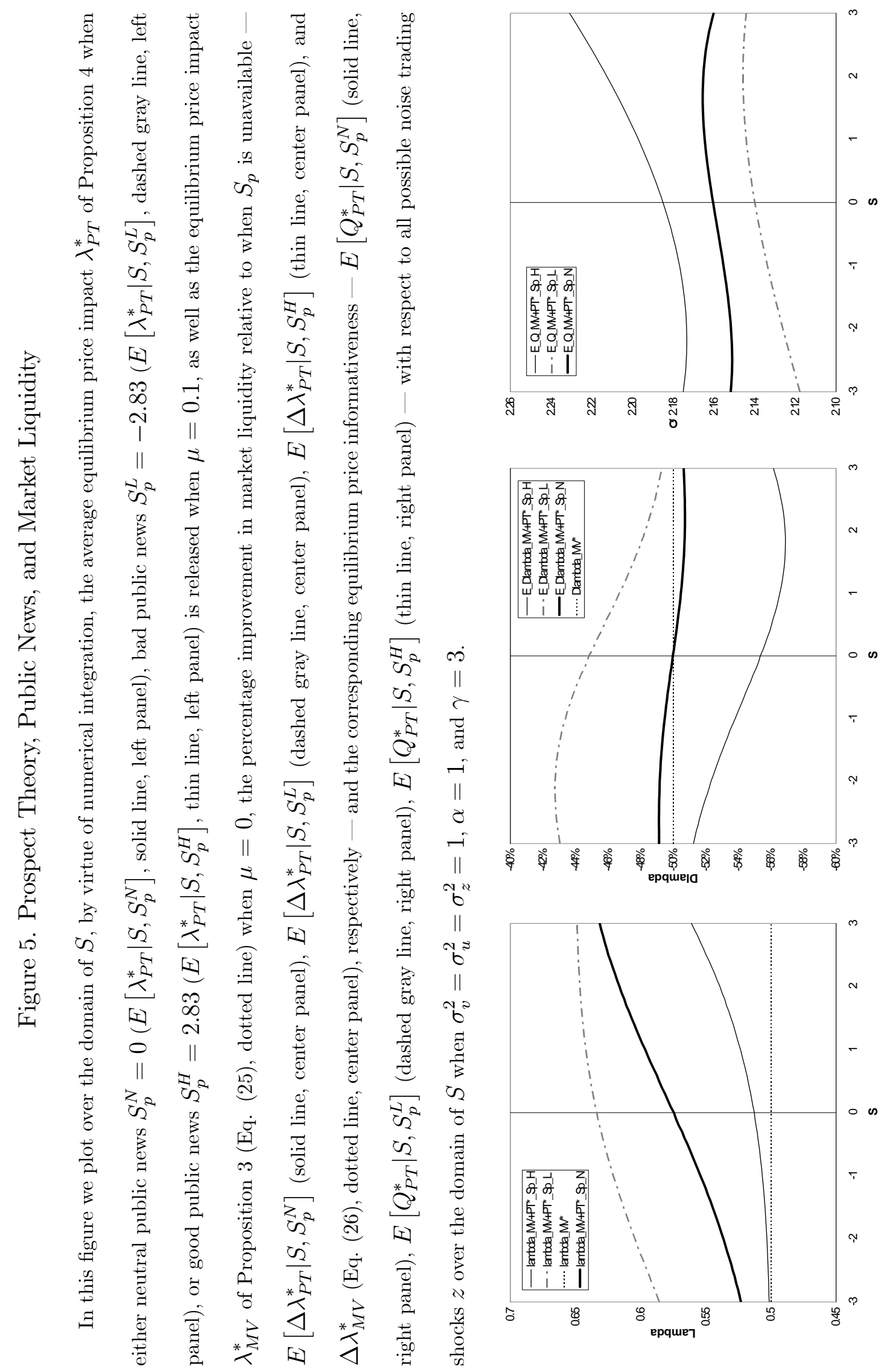

